Supporting information for

\title{
Triazolopyrimidines as a New Herbicidal Lead for Combating Weed Resistance Associated with Acetohydroxyacid Synthase Mutation
}

Yu-Chao Liu, ${ }^{\dagger}$ Ren-Yu Qu, ${ }^{\dagger}$ Qiong Chen, ${ }^{\dagger}$ Jing-Fang Yang, ${ }^{\dagger}$ Niu Cong-Wei,${ }^{\ddagger}$ Xi Zhen, ${ }^{\ddagger}, \S$ and Guang-Fu Yang ${ }^{*} \dagger, \ddagger, \S$

†Key Laboratory of Pesticide \& Chemical Biology of Ministry of Education, College of Chemistry, Central China Normal University, Wuhan 430079, PR China

$\ddagger$ State Key Laboratory of Elemento-Organic Chemistry, Nankai University, Tianjin 300071, PR China

§Collaborative Innovation Center of Chemical Science and Engineering, Tianjing 30071, PR China

*corresponding authors:

E-mail: gfyang@mail.ccnu.edu.cn (G.-F. Yang),

Tel: +86-27-67867800, Fax: +86-27-67867141. 


\section{Contents:}

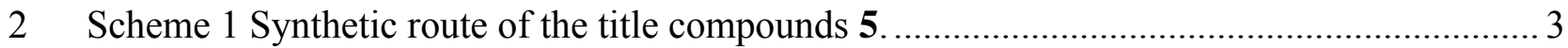

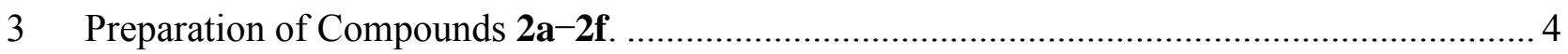

4 General Procedure for the Synthesis of Compounds 4-1-4-55 ........................................ 6

$5 \quad$ General Procedure for the Synthesis of Compounds 5-1-5-55 ......................................... 7

$6 \quad{ }^{1} \mathrm{H}$ NMR and ${ }^{13} \mathrm{C}$ NMR spectral data for representative compounds.................................. 37

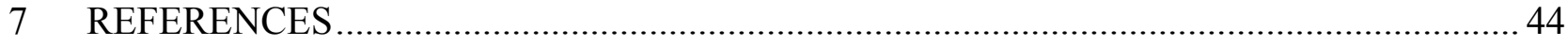

8

9

10

11

12

13

14

15

16

17

18

19

20

21

22 
Experiment details

\section{Materials and Methods}

Chemicals. 5-Methyl-2-(methylthio)-[1,2,4]triazolo[1,5-a]-pyrimidine, 5,7-dimethyl2-(methylthio)-[1,2,4]triazolo[1,5-a]-pyrimidine, 2-(methylthio)-5-phenyl-7(trifluoromethyl)-[1,2,4]-triazolo[1,5-a]pyrimidine, 5-(furan-2-yl)-2-(methylthio)-7(trifluoro-methyl)-[1,2,4]triazolo[1,5-a]pyrimidine, 2-(methylthio)-5-(thiophen-2-yl)-7(trifluoromethyl)-[1,2,4]triazolo[1,5-a]pyrimidine, 5,7-dimeth-yl-2-(methylthio)$[1,2,4]$ triazolo[1,5-c]pyrimidine, hydrogen peroxide, acetic acid, sodium hydride, toluene, the functionalized salicylates, sulfuric acid, sodium tungstate dihydrate, sodium sulfite, sodium sulfate, and water were used.

Unless otherwise noted, reagents were purchased from commercial suppliers and used without further purification because all solvents were redistilled before use. ${ }^{1} \mathrm{H}$ NMR spectra were recorded on a Mercury-Plus 400 or 600 spectrometer in $\mathrm{CDCl}_{3}$ or DMSO- $d_{6}$ with TMS as the internal reference. ${ }^{13} \mathrm{C}$ NMR spectra were recorded on a Mercury-Plus 500 (125 MHz) spectrometer in DMSO- $d_{6}$ with TMS as the internal reference. MS spectra were determined using a Trace MS 2000 organic mass spectrometer. Elemental analyses were performed on a Vario EL III elemental analysis instrument. Melting points were measured on a Buchi B-545 melting-point apparatus and are uncorrected. Intermediates 1 were prepared according to the reported methods. ${ }^{1}$ Intermediates 3 were prepared according to the reported methods. ${ }^{2-7}$

\section{Scheme 1 Synthetic route of the title compounds 5.}



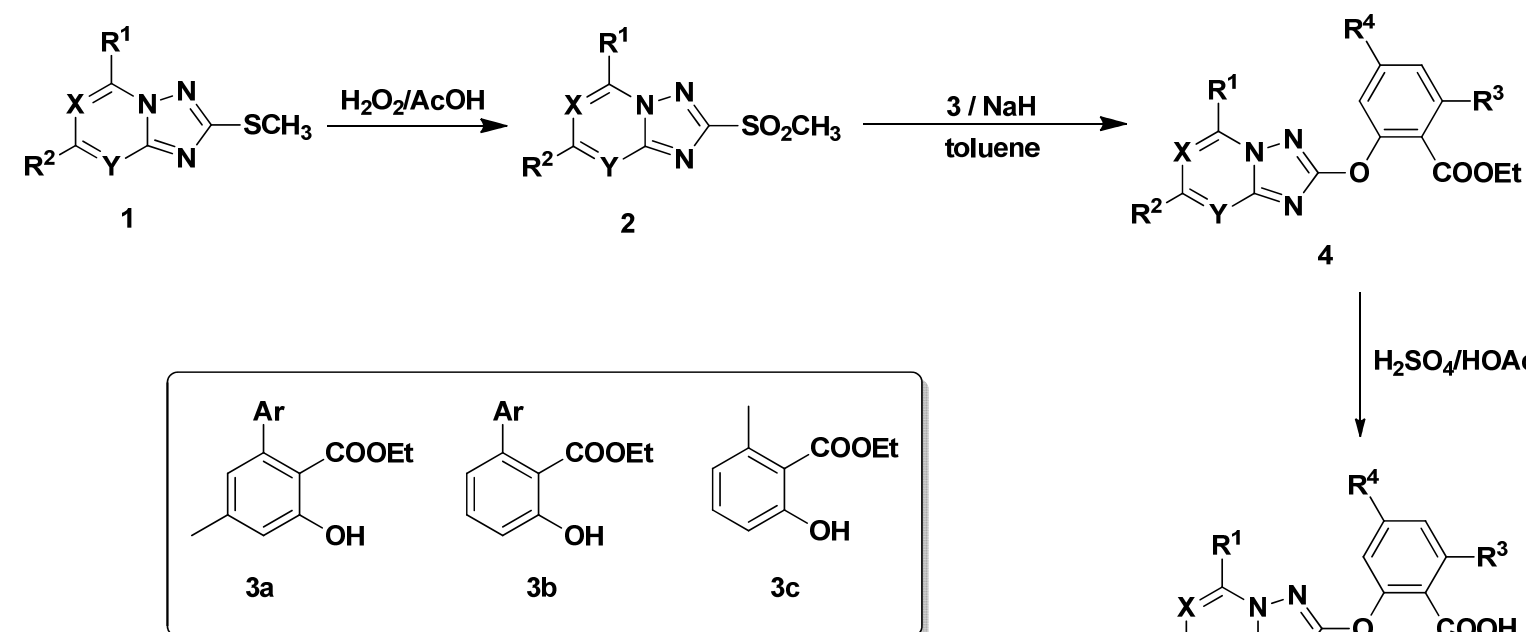

$\mathrm{H}_{2} \mathrm{SO}_{4} / \mathrm{HOAC} / \mathrm{H}_{2} \mathrm{O}$

Preparation of Compounds 2a-2f.

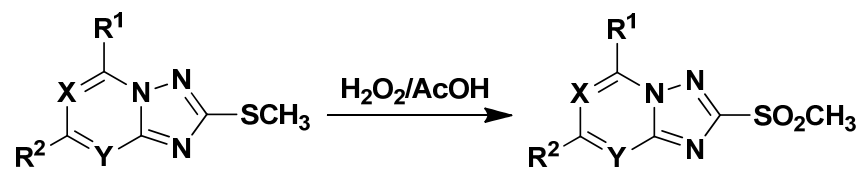

To a stirred mixture of compounds $1(0.01 \mathrm{~mol})$ and $8 \mathrm{~mL}$ of acetic acid, $0.1 \mathrm{~g}$ of $\mathrm{NaWO}_{4} \cdot \mathrm{H}_{2} \mathrm{O}$ was added at room temperature. To the vigorously stirred solution, $0.02 \mathrm{~mol}$ of hydrogen peroxide as a $30 \%$ aqueous solution was added slowly at $40{ }^{\circ} \mathrm{C}$. Stirring was continued at $50{ }^{\circ} \mathrm{C}$ for an addition $3 \mathrm{~h}$. The excess of hydrogen peroxide was destroyed by

52 the addition of an aqueous solution of sodium sulfite, and the solid was filtered off and 53 recrystallized from ethanol or purified by flash chromatography to give the pure products 2 a $54-\mathbf{2 f}$.

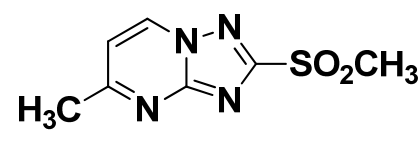


59

60

61

62

63

64 65 (m, 3H), $3.56(\mathrm{~s}, 3 \mathrm{H})$.

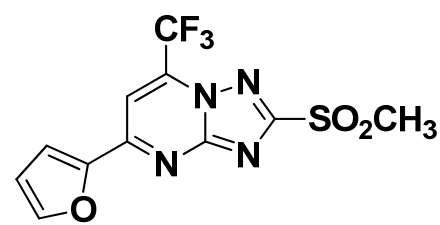

66<smiles>COS(=O)(=O)c1nc2nc(-c3ccccc3)cc(C(F)(F)F)n2n1</smiles>

2c<smiles>COS(=O)(=O)c1nc2nc(-c3cccs3)cc(C(F)(F)F)n2n1</smiles>

$2 e$

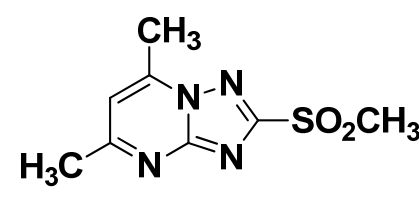

2b

Data for $\mathbf{2 b}\left(\mathrm{X}=\mathrm{CH}, \mathrm{Y}=\mathrm{N}, \mathrm{R}^{1}=\mathrm{CH}_{3}, \mathrm{R}^{2}=\mathrm{CH}_{3}\right.$ ): White solid, $85 \%$ yield, mp: $186^{-}$ $188^{\circ} \mathrm{C} .{ }^{1} \mathrm{H}$ NMR $\left(400 \mathrm{MHz}, \mathrm{CDCl}_{3} \delta\right): 6.71(\mathrm{~s}, 1 \mathrm{H}), 3.36$ (s, 3H), $2.64(\mathrm{~s}, 3 \mathrm{H}), 2.53(\mathrm{~s}, 3 \mathrm{H})$.

Data for 2c $\left(\mathrm{X}=\mathrm{CH}, \mathrm{Y}=\mathrm{N}, \mathrm{R}^{1}=\mathrm{CF}_{3}, \mathrm{R}^{2}=\mathrm{Ph}\right)$ : White solid, $82 \%$ yield, $\mathrm{mp}: 246^{-}$ $247^{\circ} \mathrm{C} .{ }^{1} \mathrm{H}$ NMR $\left(400 \mathrm{MHz}, \mathrm{DMSO}^{-}{ }_{6} \delta\right): 8.67(\mathrm{~s}, 1 \mathrm{H}), 8.42(\mathrm{~d}, J=7.2 \mathrm{~Hz}, 2 \mathrm{H}), 7.82-7.23$

Data for $2 \mathbf{d}\left(\mathrm{X}=\mathrm{CH}, \mathrm{Y}=\mathrm{N}, \mathrm{R}^{1}=\mathrm{CF}_{3}, \mathrm{R}^{2}=\right.$ furan-2-yl ): White solid, 70\% yield, mp: 227-228 ${ }^{\circ} \mathrm{C} .{ }^{1} \mathrm{H}$ NMR (400 MHz, DMSO-d 6 ) : 8.40 (d, $\left.J=10.0 \mathrm{~Hz}, 1 \mathrm{H}\right), 8.18(\mathrm{~d}, J=1.2$ Hz, 1H), 7.97 (d, $J=3.5 \mathrm{~Hz}, 1 \mathrm{H}), 6.88(\mathrm{dt}, J=6.0,3.2 \mathrm{~Hz}, 1 \mathrm{H}), 3.54(\mathrm{~s}, 3 \mathrm{H})$. 
<smiles>COS(=O)(=O)c1nc2cc(C)nc(C)n2n1</smiles>

74<smiles>[R]c1[Y]([H])c2nc(S(C)(=O)=O)nn2c1[R]</smiles><smiles>[R]c1cc([R])c(C(=O)OCC)c(Oc2nc3nc([R])[Y]([H])c([R])n3n2)c1</smiles>

78

Data for $2 f\left(X=N, Y=C H, R^{1}=\mathrm{CH}_{3}, \mathrm{R}^{2}=\mathrm{CH}_{3}\right)$ : White solid, $83 \%$ yield, mp: 193$194^{\circ} \mathrm{C} .{ }^{1} \mathrm{H}$ NMR $\left(400 \mathrm{MHz}, \mathrm{CDCl}_{3} \delta\right): 7.46$ (s, 1H), 3.40 (s, 3H), 3.03 (s, 3H), 2.64 (s, 3H).

\section{General Procedure for the Synthesis of Compounds 4-1-4-55.}<smiles>CCOC(=O)c1c(O)cc(C)cc1Br</smiles>

$3 \mathbf{a}$ then $0.002 \mathrm{~mol}$ ofcompounds 2 was added and the mixture refluxed until the reaction was complete monitored by TLC. The reaction mixture was cooled to room temperature, and<smiles>CCOC(=O)c1c(O)cccc1Cl</smiles>

3b

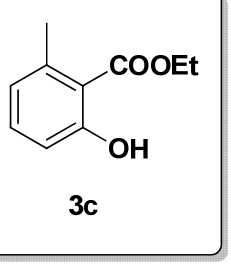

A mixture of $0.002 \mathrm{~mol}$ of the functionalized 6-arylsalicylates (3) and $0.003 \mathrm{~mol}$ of sodium hydride $(60 \%)$ in $30 \mathrm{~mL}$ of anhydrous toluene was stirred at $55-60{ }^{\circ} \mathrm{C}$ for $1 \mathrm{~h}$, and after diatomite filtration, the solvent was removed under reduced pressure to obtain crude 


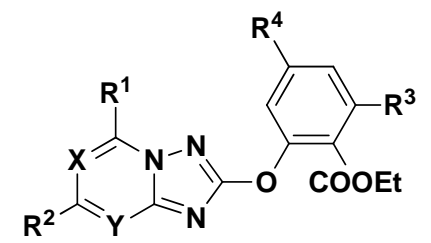

87

88

89

4

5-1

product. The crude product was purified by flash chromatography to afford the desired product or reacted on the next step directly.

\section{General Procedure for the Synthesis of Compounds 5-1-5-55.}

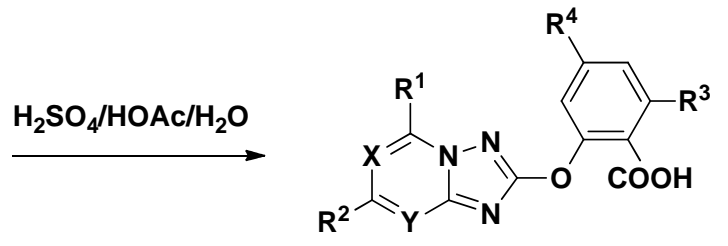

5

A total of $0.001 \mathrm{~mol}$ of compounds 4 was dissolved in the mixture solution of $\mathrm{H}_{2} \mathrm{SO}_{4}(4$ $\mathrm{mL})$, HOAc $(7 \mathrm{~mL})$, and $\mathrm{H}_{2} \mathrm{O}(1 \mathrm{~mL})$. It was then heated to reflux until the reaction was completed according to TLC detection. The reaction medium was cooled to room temperature by pouring into ice-cold water $(50 \mathrm{~mL})$. Next, the aqueous layer was extracted with $\mathrm{CH}_{2} \mathrm{Cl}_{2}(15 \mathrm{~mL})$ three times, dried by anhydrous $\mathrm{Na}_{2} \mathrm{SO}_{4}$, and then concentrated by rotary evaporation. The residue was purified via flash chromatography to give title compounds 5 in yields of $15-65 \%$.

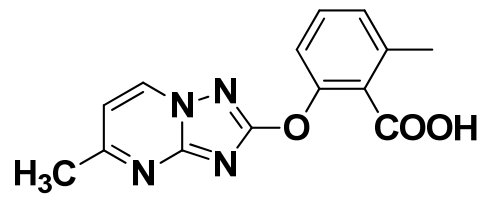

Data for 2-Methyl-6-((5-methyl-[1,2,4]triazolo[1,5-a]-pyrimidin-2-yl)oxy)benzoic Acid (5-1). White solid, $15 \%$ yield, mp: $214-215{ }^{\circ} \mathrm{C} .{ }^{1} \mathrm{H}$ NMR (600 MHz, DMSO-d $d_{6}, \delta$ ): 13.26 (s, 1H), 9.09 (d, $J=6.6 \mathrm{~Hz}, 1 \mathrm{H}), 7.40$ (t, $J=7.8 \mathrm{~Hz}, 1 \mathrm{H}), 7.25(\mathrm{~d}, J=8.4 \mathrm{~Hz}, 1 \mathrm{H}), 7.21$ (dd, $J=11.4,7.2 \mathrm{~Hz}, 2 \mathrm{H}), 2.59(\mathrm{~s}, 3 \mathrm{H}), 2.35(\mathrm{~s}, 3 \mathrm{H}) .{ }^{13} \mathrm{C} \mathrm{NMR}\left(125 \mathrm{MHz}, \mathrm{DMSO}-d_{6}, \delta\right):$ $169.52,167.38,165.17,153.46,149.91,136.25,135.92,129.96,127.61,127.08,118.73$, 
101 111.18, 24.53, 19.06. EIMS m/z: $\mathrm{M}^{+}$284.13. Anal. Calcd for $\mathrm{C}_{14} \mathrm{H}_{12} \mathrm{~N}_{4} \mathrm{O}_{3}: \mathrm{C}, 59.15 ; \mathrm{H}$,

102 4.25; N, 22319.71. Found: C, 59.38; H, 4.37; N, 19.92.

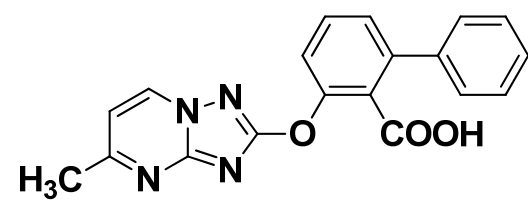

103

5-2

104

Data for 3-((5-Methyl-[1,2,4]triazolo[1,5-a]pyrimidin-2-yl)-oxy)-[1,1' 105

carboxylic Acid (5-2). White solid, 37\% yield, mp: $210-211{ }^{\circ} \mathrm{C} .{ }^{1} \mathrm{H}$ NMR $(600 \mathrm{MHz}$, 106

DMSO-d $\left._{6}, \delta\right): 13.23(\mathrm{~s}, 1 \mathrm{H}), 9.13(\mathrm{~d}, J=7.2 \mathrm{~Hz}, 1 \mathrm{H}), 7.58(\mathrm{t}, J=7.8 \mathrm{~Hz}, 1 \mathrm{H}), 7.46(\mathrm{~d}, J=$ 107

$4.2 \mathrm{~Hz}, 5 \mathrm{H}), 7.41(\mathrm{~s}, 1 \mathrm{H}), 7.34(\mathrm{~d}, J=7.8 \mathrm{~Hz}, 1 \mathrm{H}), 7.24(\mathrm{~d}, J=7.2 \mathrm{~Hz}, 1 \mathrm{H}), 2.60(\mathrm{~s}, 3 \mathrm{H})$.

108

${ }^{13} \mathrm{C}$ NMR (125 MHz, DMSO-d $\left.d_{6}, \delta\right): 169.44,167.30,165.26,153.47,149.90,140.07$, 109 $139.15,136.00,130.09,128.47,128.14,127.78,127.27,126.64,120.23,111.27,24.55$.

110 EIMS m/z: $\mathrm{M}^{+}$346.03. Anal. Calcd for $\mathrm{C}_{19} \mathrm{H}_{14} \mathrm{~N}_{4} \mathrm{O}_{3}$ : C, 65.89; H, 4.07; N, 16.18. Found: C, $111 \quad 65.90 ; \mathrm{H}, 3.99 ; \mathrm{N}, 16.31$.

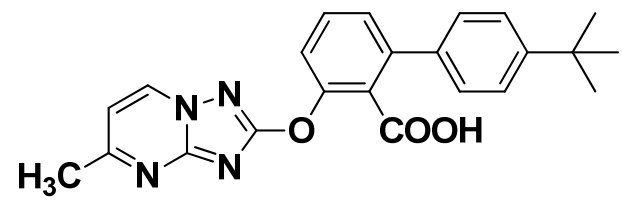

112

5-3

Data for $4^{\prime}$-(tert-Butyl)-3-((5-methyl-[1,2,4]triazolo[1,5-a]-pyrimidin-2-yl)oxy)$114\left[1,1^{\prime}\right.$-biphenyl]-2-carboxylic Acid (5-3). White solid, $18 \%$ yield, mp: $221-222{ }^{\circ} \mathrm{C} .{ }^{1} \mathrm{H}$ 115 NMR (600 MHz, DMSO- $\left.d_{6} \delta\right): 13.20(\mathrm{~s}, 1 \mathrm{H}), 9.12(\mathrm{~d}, J=7.2 \mathrm{~Hz}, 1 \mathrm{H}), 7.55(\mathrm{~d}, J=7.8 \mathrm{~Hz}$, $1161 \mathrm{H}), 7.48(\mathrm{~d}, J=8.4 \mathrm{~Hz}, 2 \mathrm{H}), 7.44(\mathrm{~d}, J=8.4 \mathrm{~Hz}, 1 \mathrm{H}), 7.40(\mathrm{~d}, J=8.4 \mathrm{~Hz}, 2 \mathrm{H}), 7.33(\mathrm{~d}, J=$ $1177.8 \mathrm{~Hz}, 1 \mathrm{H}), 7.24(\mathrm{~d}, J=7.2 \mathrm{~Hz}, 1 \mathrm{H}), 2.60(\mathrm{~s}, 3 \mathrm{H}), 1.32(\mathrm{~s}, 9 \mathrm{H}) .{ }^{13} \mathrm{C}$ NMR $(125 \mathrm{MHz}$, 118 DMSO- $_{6} \delta$ ): 169.49, 167.46, 165.26, 153.49,150.20, 149.89, 139.97, 136.27, 135.97, $119130.03,127.87,127.27,126.61,125.32,120.02,111.26,34.28,31.06,24.56$. EIMS m/z: ${ }^{+}$ 
122

123

124

125

126

127

128

129

130

131

132

133

134

135

136

137

138

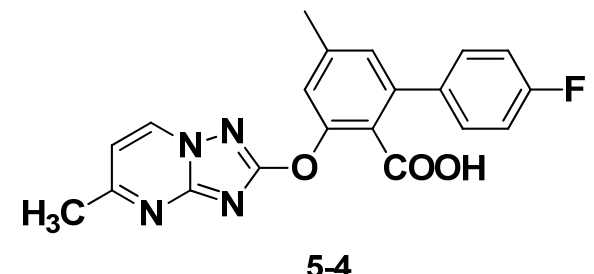

5-4

$\left[1,1^{\prime}\right.$-biphenyl]-2-carboxylic Acid (5-4). White solid, 42\% yield, mp: $215-216{ }^{\circ} \mathrm{C} .{ }^{1} \mathrm{H}$ NMR (600 MHz, DMSO-d 6 $\delta): 13.17(\mathrm{~s}, 1 \mathrm{H}), 9.14(\mathrm{~d}, J=7.2 \mathrm{~Hz}, 1 \mathrm{H}), 7.47$ (dd, $J=8.4$, $5.4 \mathrm{~Hz}, 2 \mathrm{H}), 7.33-7.26(\mathrm{~m}, 3 \mathrm{H}), 7.24(\mathrm{~d}, J=7.2 \mathrm{~Hz}, 1 \mathrm{H}), 7.17(\mathrm{~s}, 1 \mathrm{H}), 2.60$ (s, 3H), 2.38 (s, 3H). ${ }^{13} \mathrm{C}$ NMR (125 MHz, DMSO- $\left.d_{6} \delta\right): 169.47,167.37,165.23,162.83,160.89,153.48$, $150.05,140.35,138.98,135.99,135.75,135.73,130.19,130.12,127.40,124.47,120.58$, 115.37, 115.20,111.23, 24.54, 20.74. EIMS m/z: $\mathrm{M}^{+}$378.06. Anal. Calcd for $\mathrm{C}_{20} \mathrm{H}_{15} \mathrm{FN}_{4} \mathrm{O}_{3}$ : C, 63.49; H, 4.00; N, 14.81. Found: C, 63.61; H, 4.21; N, 14.98.

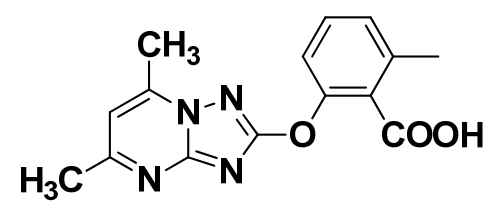

5-5

Data for 2-((5,7-Dimethyl-[1,2,4]triazolo[1,5-a]pyrimidin-2-yl)oxy)-6-methylbenzoic Acid (5-5). White solid, 17\% yield, mp: $210-211{ }^{\circ} \mathrm{C} .{ }^{1} \mathrm{H}$ NMR (600 MHz, DMSO- $\left.d_{6} \delta\right)$ : $7.39(\mathrm{t}, J=7.8 \mathrm{~Hz}, 1 \mathrm{H}), 7.26-7.10(\mathrm{~d}, J=8.4 \mathrm{~Hz}, 1 \mathrm{H}), 7.19(\mathrm{~d}, J=7.8 \mathrm{~Hz}, 1 \mathrm{H}), 7.16(\mathrm{~s}, 1 \mathrm{H})$, $4.02(\mathrm{~s}, 1 \mathrm{H}), 2.63(\mathrm{~s}, 3 \mathrm{H}), 2.54(\mathrm{~s}, 3 \mathrm{H}), 2.35(\mathrm{~s}, 3 \mathrm{H}) .{ }^{13} \mathrm{C}$ NMR $\left(125 \mathrm{MHz}\right.$, DMSO-d $\left.d_{6} \delta\right):$ $168.83,167.51,164.05,153.60,150.04,146.83,136.17,129.95,127.49,126.88,118.34$, 110.86, 24.40, 19.09, 16.46. EIMS m/z: $\mathrm{M}^{+}$298.29. Anal. Calcd for $\mathrm{C}_{15} \mathrm{H}_{14} \mathrm{~N}_{4} \mathrm{O}_{3}$ : C, 60.40; H, 4.73; N, 18.78. Found: C, 60.21; H, 4.47; N, 18.74 . 


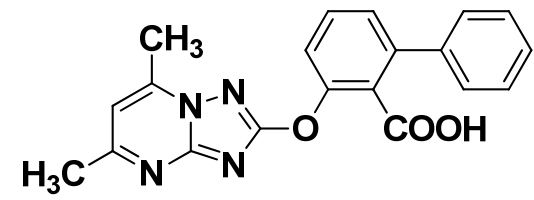

139

140

141

142

143

144

145

146

147

148

149

150

151

152

153

154

155

5-6

Data for 3-((5,7-Dimethyl-[1,2,4]triazolo[1,5-a]pyrimidin-2-yl)oxy)-[1,1' -biphenyl]2-carboxylic Acid (5-6). White solid, 57\% yield, mp: $224-225{ }^{\circ} \mathrm{C} .{ }^{1} \mathrm{H}$ NMR $(600 \mathrm{MHz}$, DMSO- $\left._{6} \delta\right): 13.23(\mathrm{~s}, 1 \mathrm{H}), 7.57(\mathrm{t}, J=7.8 \mathrm{~Hz}, 1 \mathrm{H}), 7.47(\mathrm{~d}, J=6.0 \mathrm{~Hz}, 5 \mathrm{H}), 7.41(\mathrm{~d}, J=$ $6.0 \mathrm{~Hz}, 1 \mathrm{H}), 7.33(\mathrm{~d}, J=7.8 \mathrm{~Hz}, 1 \mathrm{H}), 7.18(\mathrm{~s}, 1 \mathrm{H}), 2.65(\mathrm{~s}, 3 \mathrm{H}), 2.55(\mathrm{~s}, 3 \mathrm{H}) .{ }^{13} \mathrm{C} \mathrm{NMR}$ (125 MHz, DMSO-d $\left.d_{6} \delta\right): 168.74,167.47,164.07,153.58,150.06,146.79,140.04,139.19$, 130.05, 128.50, 128.18, 127.81, 127.11, 126.39, 119.79, 110.87, 24.37, 16.42. EIMS m/z: $\mathrm{M}^{+}$360.10. Anal. Calcd for $\mathrm{C}_{20} \mathrm{H}_{16} \mathrm{~N}_{4} \mathrm{O}_{3}$ : C, 66.66; H, 4.48; N, 15.55. Found: C, 66.46; $\mathrm{H}$, $4.59 ; \mathrm{N}, 15.32$.

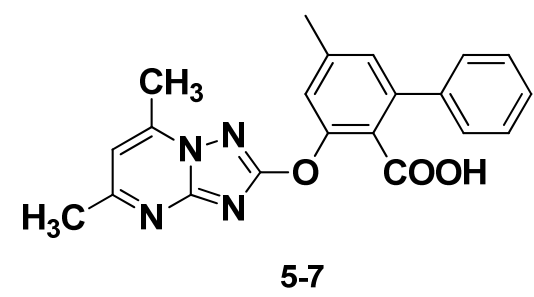

Data for 3-((5,7-Dimethyl-[1,2,4]triazolo[1,5-a]pyrimidin-2-yl)oxy)-5-methyl-[1,1 ' biphenyl]-2-carboxylic Acid (5-7). White solid, 38\% yield, mp: $230-231{ }^{\circ} \mathrm{C} .{ }^{1} \mathrm{H}$ NMR (600 MHz, DMSO-d $\left.{ }_{6} \delta\right): 13.08(\mathrm{~s}, 1 \mathrm{H}), 7.45(\mathrm{~d}, J=3.6 \mathrm{~Hz}, 4 \mathrm{H}), 7.40(\mathrm{~s}, 1 \mathrm{H}), 7.25(\mathrm{~s}, 1 \mathrm{H}), 7.18$ (s, 1H), 7.15 (s, 1H), 2.65 (s, 3H), 2.55 (s, 3H), 2.37 (s, 3H). ${ }^{13} \mathrm{C}$ NMR (125 MHz, DMSO$\left.d_{6} \delta\right): 168.91,167.35,161.20,151.48,149.89,144.89,140.07,139.11,130.08,128.48$, 128.15, 127.81, 127.05, 126.53, 119.73, 117.76, 23.76, 14.97. EIMS m/z: $\mathrm{M}^{+}$374.34. Anal. Calcd for $\mathrm{C}_{21} \mathrm{H}_{18} \mathrm{~N}_{4} \mathrm{O}_{3}$ : C, 67.37; H, 4.85; N, 14.96. Found: C, 67.65; H, 4.81; N, 14.75. 
156

157

158

159

160

161

162

163

164

165

166

167

168

169

170

171

172

173

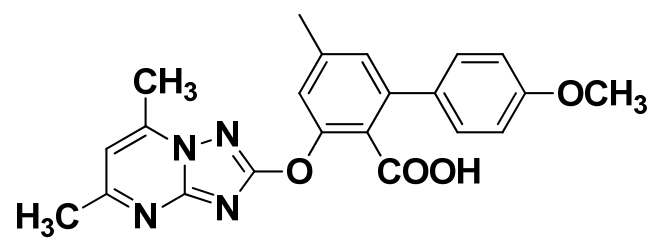

5-8

Data for 3-((5,7-Dimethyl-[1,2,4]triazolo[1,5-a]pyrimidin-2-yl)oxy)-4' -methoxy-5methyl-[1,1' -biphenyl]-2-carboxylic Acid (5-8). White solid, 18\% yield, mp: 208-209 ${ }^{\circ} \mathrm{C}$. ${ }^{1} \mathrm{H}$ NMR (600 MHz, DMSO-d $d_{6} \delta$ ): 13.04 (s, 1H), 7.39 (d, $\left.J=8.4 \mathrm{~Hz}, 2 \mathrm{H}\right), 7.20(\mathrm{~s}, 1 \mathrm{H})$, $7.18(\mathrm{~s}, 1 \mathrm{H}), 7.12(\mathrm{~s}, 1 \mathrm{H}), 7.01(\mathrm{~d}, J=8.4 \mathrm{~Hz}, 2 \mathrm{H}), 3.79(\mathrm{~s}, 3 \mathrm{H}), 2.65(\mathrm{~s}, 3 \mathrm{H}), 2.55(\mathrm{~s}, 3 \mathrm{H})$, 2.37 (s, 3H). ${ }^{13} \mathrm{C}$ NMR (125 MHz, DMSO-d 6 ): 168.76, 167.70, 163.98, 158.94, 153.56, $150.16,146.77,139.94,139.55,131.63,129.29,126.91,124.19,119.45,113.89,110.81$, 55.13, 24.35, 20.81, 16.44. EIMS m/z: $\mathrm{M}^{+}$404.06. Anal. Calcd for $\mathrm{C}_{22} \mathrm{H}_{20} \mathrm{~N}_{4} \mathrm{O}_{4}: \mathrm{C}, 65.34 ; \mathrm{H}$, 4.98; N, 13.85. Found: C, 65.44; H, 4.88; N, 13.67.

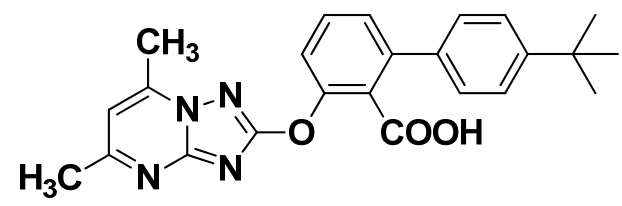

5-9

Data for 4' -(tert-Butyl)-3-((5,7-dimethyl-[1,2,4]triazolo-[1,5-a]pyrimidin-2-yl)oxy)$\left[1,1^{\prime}\right.$-biphenyl]-2-carboxylic Acid (5-9). White solid, 48\% yield, mp: $215-216{ }^{\circ} \mathrm{C} .{ }^{1} \mathrm{H}$ NMR (600 MHz, DMSO-d 6 d ): $13.20(\mathrm{~s}, 1 \mathrm{H}), 7.55$ (t, $J=7.8 \mathrm{~Hz}, 1 \mathrm{H}), 7.48(\mathrm{~d}, J=8.4 \mathrm{~Hz}$, 2H), $7.44(\mathrm{~d}, J=8.4 \mathrm{~Hz}, 1 \mathrm{H}), 7.41(\mathrm{~d}, J=8.4 \mathrm{~Hz}, 2 \mathrm{H}), 7.32(\mathrm{~d}, J=7.8 \mathrm{~Hz}, 1 \mathrm{H}), 7.18$ (s, 1H), $2.65(\mathrm{~s}, 3 \mathrm{H}), 2.55(\mathrm{~s}, 3 \mathrm{H}), 1.32(\mathrm{~s}, 9 \mathrm{H}) .{ }^{13} \mathrm{C}$ NMR (125 MHz, DMSO-d 6 $\delta$ ): 168.71, $167.50,164.05,153.57,150.17,149.96,146.83,139.83,136.28,129.90,127.87,127.12$, 126.31, 125.31, 119.50, 110.88, 34.28, 31.06, 24.37, 16.43. EIMS m/z: $\mathrm{M}^{+}$416.74. Anal. Calcd for $\mathrm{C}_{24} \mathrm{H}_{24} \mathrm{~N}_{4} \mathrm{O}_{3}$ : C, 69.21; H, 5.81; N, 13.45. Found: C, 69.23; H, 5.72; N, 13.39. 
174

175

176

177

178

179

180

181 182

183

184

185

186

187

188

189

190

191

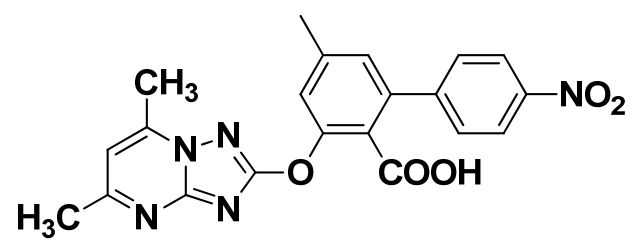

5-10

Data for 3-((5,7-Dimethyl-[1,2,4]triazolo[1,5-a]pyrimidin-2-yl)oxy)-5-methyl-4 ' nitro-[1,1' -biphenyl]-2-carboxylic Acid (5-10). White solid, 37\% yield, mp: $234-235{ }^{\circ} \mathrm{C}$. ${ }^{1} \mathrm{H}$ NMR (600 MHz, DMSO-d 6 $\delta$ ): 13.29 (s, 1H), 8.32 (d, J=8.4 Hz, 2H), 7.80-7.67 (m, 2H), $7.35(\mathrm{~s}, 1 \mathrm{H}), 7.24(\mathrm{~s}, 1 \mathrm{H}), 7.19(\mathrm{~s}, 1 \mathrm{H}), 2.65(\mathrm{~s}, 3 \mathrm{H}), 2.56(\mathrm{~s}, 3 \mathrm{H}), 2.41(\mathrm{~s}, 3 \mathrm{H}) .{ }^{13} \mathrm{C}$ NMR (125 MHz, DMSO- $\left.d_{6} \delta\right): 168.67,167.07,164.08,153.58,150.44,146.94,146.85$, $146.18,140.77,138.04,129.55,127.15,124.28,123.56,121.32,110.89,24.36,20.75,16.43$. EIMS m/z: $\mathrm{M}^{+}$419.02. Anal. Calcd for $\mathrm{C}_{21} \mathrm{H}_{17} \mathrm{~N}_{5} \mathrm{O}_{5}$ : C, 60.14; H, 4.09; N, 16.70. Found: C, $60.05 ; H, 3.94 ; \mathrm{N}, 16.87$.

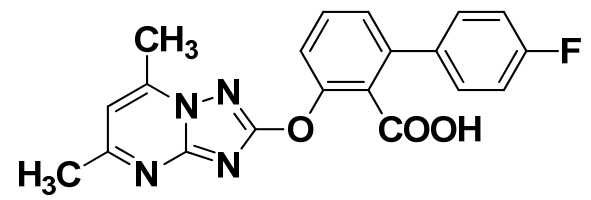

$5-11$

Data for 3-((5,7-Dimethyl-[1,2,4]triazolo[1,5-a]pyrimidin-2-yl)oxy)-4' -fluoro-[1,1' biphenyl]-2-carboxylic Acid (5-11). White solid, 41\% yield, mp: $213-214{ }^{\circ} \mathrm{C} .{ }^{1} \mathrm{H}$ NMR (600 MHz, DMSO- $\left.d_{6} \delta\right): 13.30(\mathrm{~s}, 1 \mathrm{H}), 7.57(\mathrm{~s}, 1 \mathrm{H}), 7.53-7.45(\mathrm{~m}, 3 \mathrm{H}), 7.31(\mathrm{dd}, J=14.4,7.8$ $\mathrm{Hz}, 3 \mathrm{H}), 7.18(\mathrm{~s}, 1 \mathrm{H}), 2.65(\mathrm{~s}, 3 \mathrm{H}), 2.55(\mathrm{~s}, 3 \mathrm{H}) .{ }^{13} \mathrm{C}$ NMR (125 MHz, DMSO-d $\left.d_{6} \delta\right):$ $168.66,167.32,164.09,162.90,162.08,160.96,153.58,150.02,146.85,138.92,135.57$, $135.55,130.28,130.21,130.05,126.38,119.85,115.45,115.28,110.90,24.37,16.43$. EIMS m/z: $\mathrm{M}^{+}$378.02. Anal. Calcd for $\mathrm{C}_{20} \mathrm{H}_{15} \mathrm{FN}_{4} \mathrm{O}_{3}: \mathrm{C}, 63.49 ; \mathrm{H}, 4.00 ; \mathrm{N}, 14.81$. Found: $\mathrm{C}$, $63.41 ; \mathrm{H}, 4.27 ; \mathrm{N}, 14.79$. 


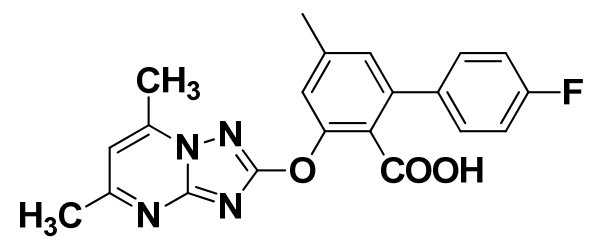

192

193

194

195

196

197

198

199

200

201

202

203

204

205

206

207

208

209

5-12

Data for 3-((5,7-Dimethyl-[1,2,4]triazolo[1,5-a]pyrimidin-2-yl)oxy)-4 ' $\quad$-fluoro-5methyl-[1,1' -biphenyl]-2-carboxylic Acid (5-12). White solid, 40\% yield, mp: $220-221{ }^{\circ} \mathrm{C}$. ${ }^{1} \mathrm{H}$ NMR (600 MHz, DMSO- $d_{6} \delta$ ): $13.13(\mathrm{~s}, 1 \mathrm{H}), 7.54-7.42(\mathrm{~m}, 2 \mathrm{H}), 7.29(\mathrm{t}, J=9.0 \mathrm{~Hz}$, 2H), $7.26(\mathrm{~s}, 1 \mathrm{H}), 7.18(\mathrm{~s}, 1 \mathrm{H}), 7.15(\mathrm{~s}, 1 \mathrm{H}), 2.65(\mathrm{~s}, 3 \mathrm{H}), 2.55(\mathrm{~s}, 3 \mathrm{H}), 2.38(\mathrm{~s}, 3 \mathrm{H}) .{ }^{13} \mathrm{C}$ NMR (125 MHz, DMSO- $\left.d_{6} \delta\right): 168.74,167.49,164.03,162.86,160.91,153.57,150.22$, $146.79,140.25,138.91,135.79,135.77,130.21,130.15,127.11,124.30,120.10,115.37$, 115.20, 110.84, 24.35, 20.77, 16.42. EIMS m/z: $\mathrm{M}^{+}$392.06. Anal. Calcd for $\mathrm{C}_{21} \mathrm{H}_{17} \mathrm{FN}_{4} \mathrm{O}_{3}$ : C, 64.28; H, 4.37; N, 14.28. Found: C, 64.06; H, 4.35; N, 14.32 .

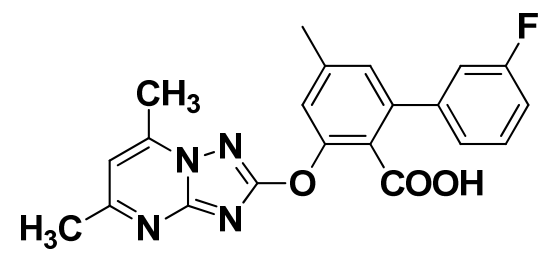

$5-13$

Data for 3-((5,7-Dimethyl-[1,2,4]triazolo[1,5-a]pyrimidin-2-yl)oxy)-3 ' -fluoro-5methyl-[1,1' -biphenyl]-2-carboxylic Acid (5-13). White solid, 40\% yield, mp: $218-219{ }^{\circ} \mathrm{C}$. ${ }^{1} \mathrm{H}$ NMR (600 MHz, DMSO- $d_{6} \delta$ ): $13.24(\mathrm{~s}, 1 \mathrm{H}), 7.51$ (dd, $\left.J=14.4,7.8 \mathrm{~Hz}, 1 \mathrm{H}\right), 7.32-$ $7.22(\mathrm{~m}, 4 \mathrm{H}), 7.19(\mathrm{~d}, J=8.4 \mathrm{~Hz}, 2 \mathrm{H}), 2.65(\mathrm{~s}, 3 \mathrm{H}), 2.55(\mathrm{~s}, 3 \mathrm{H}), 2.39(\mathrm{~s}, 3 \mathrm{H}) .{ }^{13} \mathrm{C}$ NMR (125 MHz, DMSO- $\left.d_{6} \delta\right): 168.73,167.41,164.05,162.92,160.98,153.57,150.25,146.80$, $141.75,141.68,140.43,138.53,130.50,130.43,127.09,124.42,124.41,124.26,120.55$, 115.03, 114.85, 114.63, 114.47, 110.86, 24.35, 20.77, 16.42. EIMS m/z: $\mathrm{M}^{+}$392.26. Anal. Calcd for $\mathrm{C}_{21} \mathrm{H}_{17} \mathrm{FN}_{4} \mathrm{O}_{3}$ : C, 64.28; H, 4.37; N, 14.28. Found: C, 64.04; H, 4.35; N, 14.12. 
210

211

212 213 214 215 216 217 218 219 64.17; H, 4.56; N, 14.56.

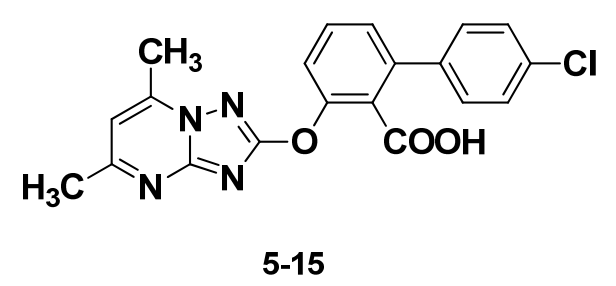

220 $\left(600 \mathrm{MHz}, \mathrm{DMSO}^{-} d_{6} \delta\right): 13.31(\mathrm{~s}, 1 \mathrm{H}), 7.58(\mathrm{t}, J=7.8 \mathrm{~Hz}, 1 \mathrm{H}), 7.54(\mathrm{~d}, J=8.4 \mathrm{~Hz}, 2 \mathrm{H})$, 224 225 226

Data for 3-((5,7-Dimethyl-[1,2,4]triazolo[1,5-a]pyrimidin-2-yl)oxy)-2 ' -fluoro-5methyl-[1,1' -biphenyl]-2-carboxylic Acid (5-14). White solid, 32\% yield, mp: $210-21{ }^{\circ} \mathrm{C}$. ${ }^{1} \mathrm{H}$ NMR (600 MHz, DMSO- $d_{6} \delta$ ): $12.91(\mathrm{~s}, 1 \mathrm{H}), 7.47-7.41(\mathrm{~m}, 1 \mathrm{H}), 7.39(\mathrm{t}, J=7.8 \mathrm{~Hz}$, 1H), $7.29(\mathrm{~s}, 1 \mathrm{H}), 7.27(\mathrm{dd}, J=8.4,4.5 \mathrm{~Hz}, 2 \mathrm{H}), 7.18(\mathrm{~s}, 1 \mathrm{H}), 7.13(\mathrm{~s}, 1 \mathrm{H}), 2.65(\mathrm{~s}, 3 \mathrm{H}), 2.55$ (s, 3H), $2.38(\mathrm{~s}, 3 \mathrm{H}) .{ }^{13} \mathrm{C}$ NMR (125 MHz, DMSO-d 6 $\left.\delta\right): 168.79,166.65,163.96,159.87$, $157.92,153.56,150.51,146.77,140.53,134.65,131.01,129.96,129.90,128.09,127.15$, $127.03,124.63,124.29,124.27,121.06,115.49,115.31,110.80,24.35,20.70,16.43$. EIMS m/z: $\mathrm{M}^{+}$392.11. Anal. Calcd for $\mathrm{C}_{21} \mathrm{H}_{17} \mathrm{FN}_{4} \mathrm{O}_{3}$ : C, 64.28; H, 4.37; N, 14.28. Found: C,

Data for 4' -Chloro-3-((5,7-dimethyl-[1,2,4]triazolo[1,5-a]-pyrimidin-2-yl)oxy)-[1,1' -biphenyl]-2-carboxylic Acid (5-15). White solid, 60\% yield, mp: $211-212{ }^{\circ} \mathrm{C} .{ }^{1} \mathrm{H}$ NMR $\left(600 \mathrm{MHz}, \mathrm{DMSO}-d_{6} \delta\right): 13.31(\mathrm{~s}, 1 \mathrm{H}), 7.58(\mathrm{t}, J=7.8 \mathrm{~Hz}, 1 \mathrm{H}), 7.54(\mathrm{~d}, J=8.4 \mathrm{~Hz}, 2 \mathrm{H})$, $7.51-7.45(\mathrm{~m}, 3 \mathrm{H}), 7.33(\mathrm{~d}, J=7.2 \mathrm{~Hz}, 1 \mathrm{H}), 7.18(\mathrm{~s}, 1 \mathrm{H}), 2.65(\mathrm{~s}, 3 \mathrm{H}), 2.55(\mathrm{~s}, 3 \mathrm{H}) .{ }^{13} \mathrm{C}$ NMR (125 MHz, DMSO- $\left.d_{6} \delta\right): 168.66,167.27,164.08,153.56,150.10,146.80,138.77$, $138.00,132.86,130.22,129.99,128.51,126.99,126.32,120.16,110.88,24.36,16.42$

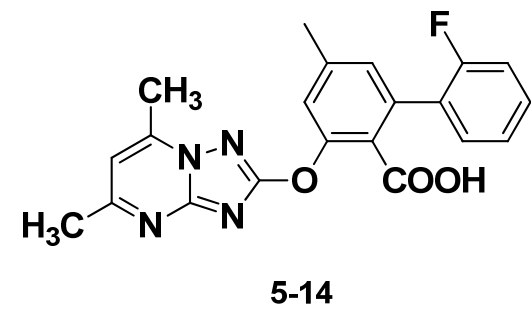



C, 60.78; H, 3.92; N, 14.34 .

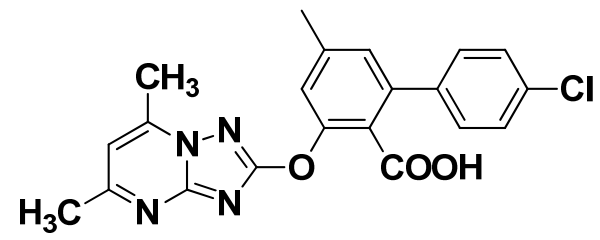

229

230

231

232

233

234

235

236

237

238

239

240

241

242

243

244

$5-16$

Data for $4{ }^{\prime}$-Chloro-3-((5,7-dimethyl-[1,2,4]triazolo[1,5-a]-pyrimidin-2-yl)oxy)-5methyl-[1,1' -biphenyl]-2-carboxylic Acid (5-16). White solid, 42\% yield, mp: $216-217^{\circ} \mathrm{C}$. ${ }^{1} \mathrm{H}$ NMR (600 MHz, DMSO-d $d_{6} \delta$ ): $13.15(\mathrm{~s}, 1 \mathrm{H}), 7.52(\mathrm{~d}, J=8.4 \mathrm{~Hz}, 2 \mathrm{H}), 7.46(\mathrm{~d}, J=8.4$ $\mathrm{Hz}, 2 \mathrm{H}), 7.27(\mathrm{~s}, 1 \mathrm{H}), 7.18(\mathrm{~s}, 1 \mathrm{H}), 7.16(\mathrm{~s}, 1 \mathrm{H}), 2.65(\mathrm{~s}, 3 \mathrm{H}), 2.55(\mathrm{~s}, 3 \mathrm{H}), 2.38(\mathrm{~s}, 3 \mathrm{H}) .{ }^{13} \mathrm{C}$ NMR (125 MHz, DMSO-d $\left.d_{6} \delta\right): 168.73,167.40,164.02,153.56,150.29,146.76,140.41$, $138.74,138.24,132.72,129.94,128.43,127.02,124.20,120.39,110.82,24.35,20.78,16.42$. EIMS m/z: $\mathrm{M}^{+}$408.80. Anal. Calcd for $\mathrm{C}_{21} \mathrm{H}_{17} \mathrm{ClN}_{4} \mathrm{O}_{3}$ : C, 61.69; H, 4.19; N, 13.70. Found: C, $61.82 ; \mathrm{H}, 4.23 ; \mathrm{N}, 13.54$.

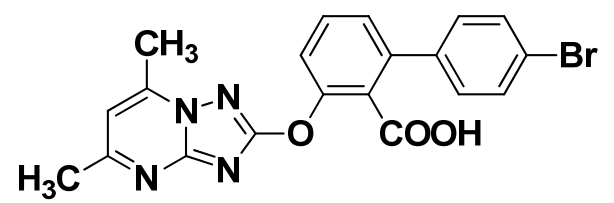

5-17

Data for 4' -Bromo-3-((5,7-dimethyl-[1,2,4]triazolo[1,5-a]-pyrimidin-2-yl)oxy)-[1,1' -biphenyl]-2-carboxylic Acid (5-17). White solid, 33\% yield, mp: $219-220{ }^{\circ} \mathrm{C} .{ }^{1} \mathrm{H}$ NMR $\left(600 \mathrm{MHz}, \mathrm{DMSO}_{6} \delta\right): 13.32(\mathrm{~s}, 1 \mathrm{H}), 7.67(\mathrm{~d}, J=8.4 \mathrm{~Hz}, 2 \mathrm{H}), 7.58(\mathrm{t}, J=7.8 \mathrm{~Hz}, 1 \mathrm{H})$, $7.49(\mathrm{~d}, J=8.4 \mathrm{~Hz}, 1 \mathrm{H}), 7.42(\mathrm{~d}, J=8.4 \mathrm{~Hz}, 2 \mathrm{H}), 7.33(\mathrm{~d}, J=7.8 \mathrm{~Hz}, 1 \mathrm{H}), 7.18(\mathrm{~s}, 1 \mathrm{H})$, $2.65(\mathrm{~s}, 3 \mathrm{H}), 2.55(\mathrm{~s}, 3 \mathrm{H}) .{ }^{13} \mathrm{C}$ NMR $\left(125 \mathrm{MHz}, \mathrm{DMSO}-d_{6} \delta\right): 168.64,167.25,164.09$, $153.56,150.08,146.84,138.77,138.39,131.43,130.31,130.19,126.98,126.24,121.43$, 
120.15, 110.90, 24.37, 16.43. EIMS m/z: $\mathrm{M}^{+}$439.03. Anal. Calcd for $\mathrm{C}_{20} \mathrm{H}_{15} \mathrm{BrN}_{4} \mathrm{O}_{3}: \mathrm{C}$,

$24654.69 ; \mathrm{H}, 3.44 ; \mathrm{N}, 12.75$. Found: C, 54.86; H, 3.64; N, 12.58.

247

248

249

250

251

252

253

254

255

256

257

258

259

260

261

262

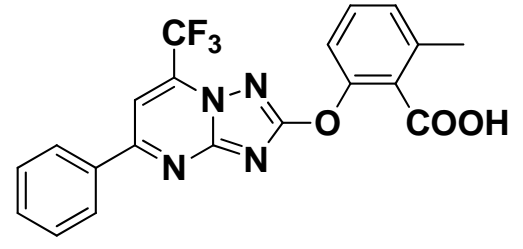

5-18

Data for 2-Methyl-6-((5-phenyl-7-(trifluoromethyl)-[1,2,4]-triazolo[1,5-a]pyrimidin-2-

yl)oxy)benzoic Acid (5-18). White solid, 61\% yield, mp: $211-212{ }^{\circ} \mathrm{C} .{ }^{1} \mathrm{H}$ NMR $(600 \mathrm{MHz}$, $\left.\operatorname{DMSO}_{-} d_{6} \delta\right): 13.37(\mathrm{~s}, 1 \mathrm{H}), 8.43(\mathrm{~s}, 1 \mathrm{H}), 8.37(\mathrm{~d}, J=7.2 \mathrm{~Hz}, 2 \mathrm{H}), 7.68-7.57(\mathrm{~m}, 3 \mathrm{H}), 7.45$ $(\mathrm{t}, J=7.8 \mathrm{~Hz}, 1 \mathrm{H}), 7.31(\mathrm{~d}, J=8.4 \mathrm{~Hz}, 1 \mathrm{H}), 7.24(\mathrm{~d}, J=7.8 \mathrm{~Hz}, 1 \mathrm{H}), 2.38(\mathrm{~s}, 3 \mathrm{H}) .{ }^{13} \mathrm{C}$ NMR (125 MHz, DMSO-d $6 \delta$ ): 170.13, 167.34, 160.70, 154.54, 149.75, 136.36, 134.98, $134.26,133.95,133.65,133.34,132.07,130.01,129.15,127.87,127.51,127.29,122.08$, 119.90, 118.43, 117.71, 115.53, 106.29, 19.08. EIMS m/z: $\mathrm{M}^{+}$414.15. Anal. Calcd for $\mathrm{C}_{20} \mathrm{H}_{13} \mathrm{~F}_{3} \mathrm{~N}_{4} \mathrm{O}_{3}$ : C, 57.98; H, 3.16; N, 13.52. Found: C, 57.79; H, 3.26; N, 13.30.

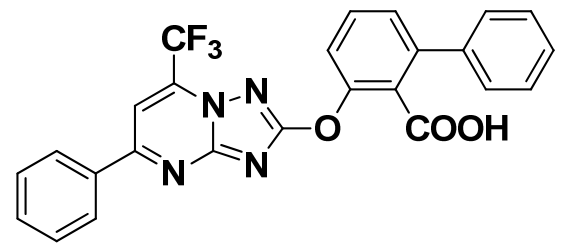

5-19

Data for 3-((5-Phenyl-7-(trifluoromethyl)-[1,2,4]triazolo-[1,5-a]pyrimidin-2-yl)oxy)$\left[1,1^{\prime}\right.$-biphenyl]-2-carboxylic Acid (5-19). White solid, 50\% yield, mp: $236-237{ }^{\circ} \mathrm{C} .{ }^{1} \mathrm{H}$ NMR (600 MHz, DMSO- $\left.d_{6} \delta\right): 13.37(\mathrm{~s}, 1 \mathrm{H}), 8.47(\mathrm{~s}, 1 \mathrm{H}), 8.39(\mathrm{~d}, J=7.2 \mathrm{~Hz}, 2 \mathrm{H}), 7.63$ (d, $J=7.2 \mathrm{~Hz}, 4 \mathrm{H}), 7.53(\mathrm{~d}, J=8.4 \mathrm{~Hz}, 1 \mathrm{H}), 7.48(\mathrm{~d}, J=7.2 \mathrm{~Hz}, 4 \mathrm{H}), 7.42$ (s, 1H), 7.39 (d, $J=7.8 \mathrm{~Hz}, 1 \mathrm{H}) .{ }^{13} \mathrm{C}$ NMR $\left(125 \mathrm{MHz}, \mathrm{DMSO}^{-} d_{6} \delta\right): 170.01,167.29,160.80,154.57$, $149.76,140.13,139.10,135.01,134.33,134.02,133.72,133.41,132.14,130.16,129.20$, 
EIMS m/z: $\mathrm{M}^{+}$476.33. Anal. Calcd for $\mathrm{C}_{25} \mathrm{H}_{15} \mathrm{~F}_{3} \mathrm{~N}_{4} \mathrm{O}_{3}$ : $\mathrm{C}, 63.03 ; \mathrm{H}, 3.17 ; \mathrm{N}, 11.76$. Found:

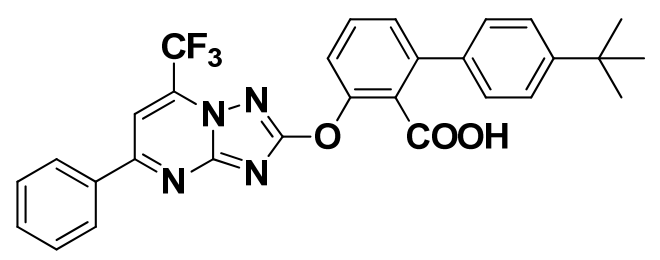

266

5-20

Data for 4 ' -(tert-Butyl)-3-((5-phenyl-7-(trifluoromethyl)-[1,2,4]triazolo[1,5268

a]pyrimidin-2-yl)oxy)-[1,1' -biphenyl]-2-carboxylic Acid (5-20). White solid, 16\% yield, 269

mp: $226-227^{\circ} \mathrm{C} .{ }^{1} \mathrm{H}$ NMR (600 MHz, DMSO-d 6 $\delta$ ): $13.32(\mathrm{~s}, 1 \mathrm{H}), 8.46(\mathrm{~s}, 1 \mathrm{H}), 8.38(\mathrm{~d}, J$ 270 $=7.2 \mathrm{~Hz}, 2 \mathrm{H}), 7.62(\mathrm{dt}, J=12.6,7.2 \mathrm{~Hz}, 4 \mathrm{H}), 7.54-7.47(\mathrm{~m}, 3 \mathrm{H}), 7.43(\mathrm{~d}, J=8.4 \mathrm{~Hz}, 2 \mathrm{H})$,

271

272

273

274 275 10.52. Found: C, 65.36; H, 4.36; N, 10.68 .

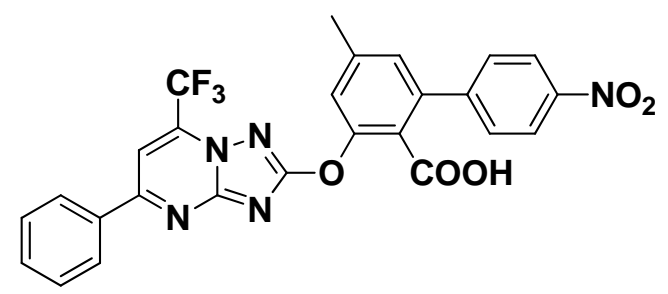

276 mp: $213-214{ }^{\circ} \mathrm{C} .{ }^{1} \mathrm{H}$ NMR $\left(600 \mathrm{MHz}, \mathrm{DMSO}_{6} \delta\right): 13.42(\mathrm{~s}, 1 \mathrm{H}), 8.48(\mathrm{~s}, 1 \mathrm{H}), 8.39$ (d, $J$ 280

$7.38(\mathrm{~d}, J=7.8 \mathrm{~Hz}, 1 \mathrm{H}), 1.32(\mathrm{~s}, 9 \mathrm{H}) .{ }^{13} \mathrm{C}$ NMR (125 MHz, DMSO- $\left.d_{6} \delta\right): 170.05,167.41$, $160.77,154.55,150.26,149.72,140.03,136.19,134.96,134.01,133.70,132.10,130.10$, $129.15,127.88,127.11,126.78,125.35,122.10,119.91,119.66,117.73,115.50,106.40$, 34.29, 31.05. EIMS m/z: $\mathrm{M}^{+}$532.04. Anal. Calcd for $\mathrm{C}_{29} \mathrm{H}_{23} \mathrm{~F}_{3} \mathrm{~N}_{4} \mathrm{O}_{3}: \mathrm{C}, 65.41 ; \mathrm{H}, 4.35 ; \mathrm{N}$, 5-21

Data for 5-Methyl-4 ' -nitro-3-((5-phenyl-7-(trifluorometh-yl)-[1,2,4]triazolo[1,5a]pyrimidin-2-yl)oxy)-[1,1' -biphenyl]-2-carboxylic Acid (5-21). White solid, 61\% yield, $=7.2 \mathrm{~Hz}, 2 \mathrm{H}), 8.33(\mathrm{~d}, J=9.0 \mathrm{~Hz}, 2 \mathrm{H}), 7.74(\mathrm{~d}, J=8.4 \mathrm{~Hz}, 2 \mathrm{H}), 7.64(\mathrm{dd}, J=10.8,7.2 \mathrm{~Hz}$, 
281 282 283 284 285

286 287 288 289 290 291 292 293 294 295 $2.85 ; \mathrm{N}, 11.33$. Found: $\mathrm{C}, 60.45 ; \mathrm{H}, 3.07 ; \mathrm{N}, 11.12$.

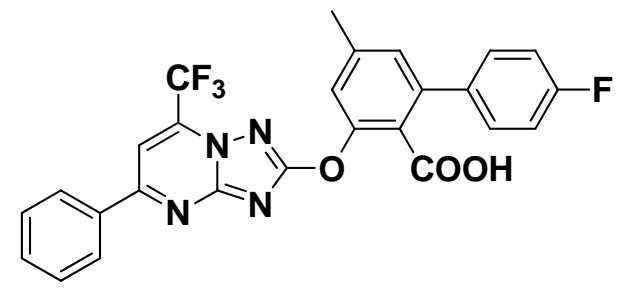

296

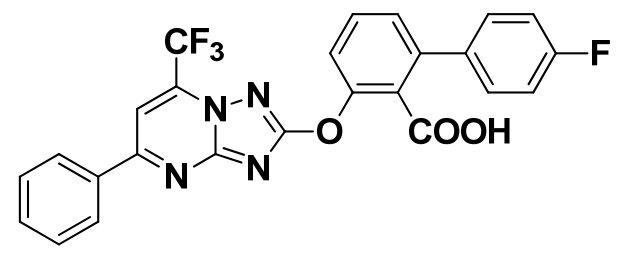

5-22

$5-23$

3H), $7.43(\mathrm{~s}, 1 \mathrm{H}), 7.31(\mathrm{~s}, 1 \mathrm{H}), 2.43(\mathrm{~s}, 3 \mathrm{H}) .{ }^{13} \mathrm{C}$ NMR (125 MHz, DMSO-d 6 ) : 170.02, $166.95,160.80,154.56,150.20,146.98,146.06,141.04,138.25,134.95,134.32,134.02$, $133.72,133.41,132.13,129.56,129.17,127.89,127.63,124.20,123.58,122.09,121.52$ 119.91, 117.72, 115.54, 106.43, 20.79. EIMS m/z: $\mathrm{M}^{+}$535.36. Anal. Calcd for $\mathrm{C}_{26} \mathrm{H}_{16} \mathrm{~F}_{3} \mathrm{~N}_{5} \mathrm{O}_{5}: \mathrm{C}, 58.32 ; \mathrm{H}, 3.01 ; \mathrm{N}, 13.08$. Found: $\mathrm{C}, 58.41 ; \mathrm{H}, 3.10 ; \mathrm{N}, 12.87$.

Data for 4' -Fluoro-3-((5-phenyl-7-(trifluoromethyl)-[1,2,4]triazolo[1,5-a]pyrimidin2-yl)oxy)-[1,1' -biphenyl]-2-carboxylic Acid (5-22). White solid, 38\% yield, mp: 218$219{ }^{\circ} \mathrm{C} .{ }^{1} \mathrm{H}$ NMR $\left(600 \mathrm{MHz}, \mathrm{DMSO}_{6} \delta\right.$ ): $13.38(\mathrm{~s}, 1 \mathrm{H}), 8.46(\mathrm{~s}, 1 \mathrm{H}), 8.38(\mathrm{~d}, J=7.2 \mathrm{~Hz}$, 2H), $7.63(\mathrm{dt}, J=7.8,5.4 \mathrm{~Hz}, 4 \mathrm{H}), 7.56^{-7.50}(\mathrm{~m}, 3 \mathrm{H}), 7.38(\mathrm{~d}, J=7.8 \mathrm{~Hz}, 1 \mathrm{H}), 7.32(\mathrm{t}, J=$ $9.0 \mathrm{~Hz}, 2 \mathrm{H}) .{ }^{13} \mathrm{C}$ NMR (125 MHz, DMSO-d $6 \delta$ ): 169.94, 167.20, 162.93, 160.99, 160.81, $154.56,149.75,139.08,135.45,135.43,134.99,134.33,134.02,133.72,133.45,132.14$, $130.29,130.22,129.19,127.91,127.11,126.83,122.09,119.98,119.90,117.72,115.49$, 115.32, 106.46, 99.47. EIMS m/z: $\mathrm{M}^{+}$494.14. Anal. Calcd for $\mathrm{C}_{25} \mathrm{H}_{14} \mathrm{~F}_{4} \mathrm{~N}_{4} \mathrm{O}_{3}: \mathrm{C}, 60.73 ; \mathrm{H}$,

Data for 4' -Fluoro-5-methyl-3-((5-phenyl-7-(trifluoro-methyl)-[1,2,4]triazolo[1,5a]pyrimidin-2-yl)oxy)-[1,1' -bi-phenyl]-2-carboxylic Acid (5-23). White solid, 60\% yield, 
mp: $220-221{ }^{\circ} \mathrm{C} .{ }^{1} \mathrm{H}$ NMR (400 MHz, DMSO-d6 $\delta$ ): 13.21 (s, 1H), 8.45 (s, 1H), 8.38 (d, J $300=7.8 \mathrm{~Hz}, 2 \mathrm{H}), 7.63(\mathrm{~d}, J=7.2 \mathrm{~Hz}, 3 \mathrm{H}), 7.50(\mathrm{dd}, J=8.45 .4 \mathrm{~Hz}, 2 \mathrm{H}), 7.35-7.27(\mathrm{~m}, 3 \mathrm{H})$, $3017.20(\mathrm{~s}, 1 \mathrm{H}), 2.41(\mathrm{~s}, 3 \mathrm{H}) .{ }^{13} \mathrm{C}$ NMR $\left(125 \mathrm{MHz}, \mathrm{DMSO}-d_{6} \delta\right): 170.02,168.92,167.31$, 302 $162.86,160.91,160.75,154.55,149.91,140.35,138.98,135.69,135.00,134.00,133.67$, 303 $132.14,130.21,130.14,129.20,127.91,127.53,126.18,120.20,119.91,117.70,117.35$, $304115.39,115.22,115.12,106.42,20.79$. EIMS m/z: $\mathrm{M}^{+}$507.98. Anal. Calcd for 305

$\mathrm{C}_{26} \mathrm{H}_{16} \mathrm{~F}_{4} \mathrm{~N}_{4} \mathrm{O}_{3}$ : C, 61.42; H, 3.17; N, 11.02. Found: C, 61.32; H, 3.35; N, 10.89.

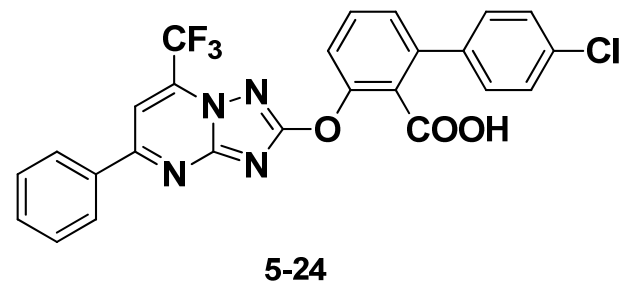

306

307

Data for 4' -Chloro-3-((5-phenyl-7-(trifluoromethyl)-[1,2,4]triazolo[1,5-a]pyrimidin308

2-yl)oxy)-[1,1' -biphenyl]-2-carboxylic Acid (5-24). White solid, 47\% yield, mp: 232309

$233{ }^{\circ} \mathrm{C} .{ }^{1} \mathrm{H}$ NMR $(600 \mathrm{MHz}, \mathrm{DMSO}-d 6 \delta$ ): 13.43 (s, 1H), $8.46(\mathrm{~s}, 1 \mathrm{H}), 8.38(\mathrm{~d}, J=7.2 \mathrm{~Hz}$, 310 2H), 7.70-7.57 (m, 4H), $7.55(\mathrm{~d}, J=8.4 \mathrm{~Hz}, 3 \mathrm{H}), 7.50(\mathrm{~d}, J=8.4 \mathrm{~Hz}, 2 \mathrm{H}), 7.39(\mathrm{~s}, 1 \mathrm{H}) .{ }^{13} \mathrm{C}$ 311 NMR (125 MHz, DMSO- $\left.d_{6} \delta\right): 169.95,167.12,160.81,154.55,149.80,138.88,137.92$, $312134.98,134.02,133.71,132.88,132.13,130.39,130.33,130.00,129.18,128.53,127.91$, $313126.75,122.09,120.25,119.87,117.71,106.45$. EIMS m/z: $\mathrm{M}^{+}$510.00. Anal. Calcd for $314 \mathrm{C}_{25} \mathrm{H}_{14} \mathrm{ClF}_{3} \mathrm{~N}_{4} \mathrm{O}_{3}:$ C, 58.78; H, 2.76; N, 10.97. Found: C, 58.69; H, 2.72; N, 10.82.

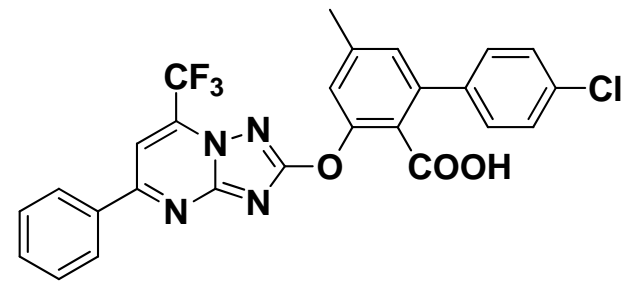

5-25 
317 a]pyrimidin-2-yl)oxy)-[1,1' -bi-phenyl]-2-carboxylic Acid (5-25). White solid, 38\% yield, 318 mp: $218-219{ }^{\circ} \mathrm{C} .{ }^{1} \mathrm{H}$ NMR $\left(600 \mathrm{MHz}, \mathrm{DMSO}^{-} d_{6} \delta\right): 13.23$ (s, 1H), 8.45 (s, 1H), 8.38 (d, J $319=7.2 \mathrm{~Hz}, 2 \mathrm{H}), 7.63(\mathrm{dd}, J=10.8,7.2 \mathrm{~Hz}, 3 \mathrm{H}), 7.53(\mathrm{~d}, J=8.4 \mathrm{~Hz}, 2 \mathrm{H}), 7.48(\mathrm{~d}, J=8.4 \mathrm{~Hz}$, 320 2H), $7.34(\mathrm{~s}, 1 \mathrm{H}), 7.21(\mathrm{~s}, 1 \mathrm{H}), 2.41(\mathrm{~s}, 3 \mathrm{H}) .{ }^{13} \mathrm{C}$ NMR $\left(125 \mathrm{MHz}, \mathrm{DMSO}-d_{6} \delta\right): 170.02$, 321 $167.23,160.77,154.56,150.01,140.57,138.86,138.15,134.99,134.01,133.71,133.40$, 322 $132.76,132.14,131.52,129.95,129.20,128.47,127.91,127.47,124.24,122.10,120.50$,

$\mathrm{C}_{26} \mathrm{H}_{16} \mathrm{ClF}_{3} \mathrm{~N}_{4} \mathrm{O}_{3}:$ C, 59.50; H, 3.07; N, 10.67. Found: C, 59.41; H, 3.18; N, 10.43.

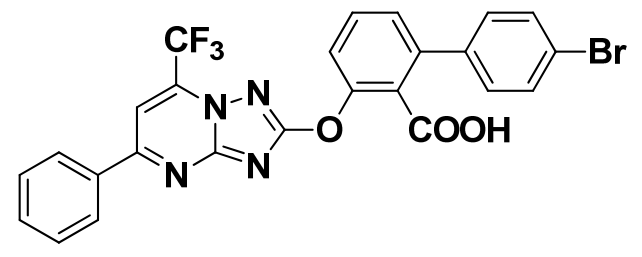

5-26

Data for 4' -Bromo-3-((5-phenyl-7-(trifluoromethyl)-[1,2,4]triazolo[1,5-a]pyrimidin-

2-yl)oxy)-[1,1' -biphenyl]-2-carboxylic Acid (5-26). White solid, 56\% yield, mp: 216328

$217{ }^{\circ} \mathrm{C} .{ }^{1} \mathrm{H}$ NMR $\left(600 \mathrm{MHz}, \mathrm{DMSO}^{-} d_{6} \delta\right.$ ): 13.42 (s, 1H), $8.46(\mathrm{~s}, 1 \mathrm{H}), 8.38$ (d, J=7.2 Hz, 329 2H), $7.68(\mathrm{~d}, J=8.4 \mathrm{~Hz}, 2 \mathrm{H}), 7.63(\mathrm{dt}, J=11.4,6.6 \mathrm{~Hz}, 4 \mathrm{H}), 7.55(\mathrm{~d}, J=8.4 \mathrm{~Hz}, 1 \mathrm{H}), 7.43$ $330(\mathrm{~d}, J=8.4 \mathrm{~Hz}, 2 \mathrm{H}), 7.39(\mathrm{~d}, J=7.8 \mathrm{~Hz}, 1 \mathrm{H}) .{ }^{13} \mathrm{C}$ NMR $\left(125 \mathrm{MHz}, \mathrm{DMSO}_{6} \delta\right.$ ): 170.27, $331169.97,167.09,160.81,154.55,149.83,138.96,138.28,134.99,134.93,134.32,134.02$, $332133.71,133.42,132.12,131.46,130.39,130.30,129.18,127.91,126.99,126.71,122.08$, 333 121.51, 120.30, 119.89, 117.71, 106.44. EIMS m/z: $\mathrm{M}^{+}$555.64. Anal. Calcd for $334 \mathrm{C}_{25} \mathrm{H}_{14} \mathrm{BrF}_{3} \mathrm{~N}_{4} \mathrm{O}_{3}: \mathrm{C}, 54.07 ; \mathrm{H}, 2.54 ; \mathrm{N}, 10.09$. Found: $\mathrm{C}, 54.02 ; \mathrm{H}, 2.57 ; \mathrm{N}, 9.87$. 


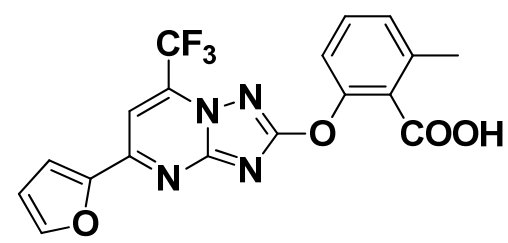

335

336

337

338

339

340

345

346

347

348

349

350

351

352

$5-27$

Data for 2-((5-(Furan-2-yl)-7-(trifluoromethyl)-[1,2,4]-triazolo[1,5-a]pyrimidin-2yl)oxy)-6-methylbenzoic Acid (5-27). Yellow solid, 62\% yield, mp: $218-219{ }^{\circ} \mathrm{C} .{ }^{1} \mathrm{H}$ NMR $\left(600 \mathrm{MHz}, \mathrm{DMSO}_{6} \delta\right.$ ): $13.35(\mathrm{~s}, 1 \mathrm{H}), 8.12(\mathrm{~d}, J=3.6 \mathrm{~Hz}, 2 \mathrm{H}), 7.78(\mathrm{~d}, J=3.6 \mathrm{~Hz}, 1 \mathrm{H})$, $7.43(\mathrm{t}, J=8.4 \mathrm{~Hz}, 1 \mathrm{H}), 7.29(\mathrm{~d}, J=8.4 \mathrm{~Hz}, 1 \mathrm{H}), 7.23(\mathrm{~d}, J=7.8 \mathrm{~Hz}, 1 \mathrm{H}), 6.85(\mathrm{~d}, J=1.8$ $\mathrm{Hz}, 1 \mathrm{H}), 2.37$ (s, 3H). ${ }^{13} \mathrm{C}$ NMR (125 MHz, DMSO- $\left.d_{6} \delta\right): 169.99,167.37,154.55,151.48$, $150.00,149.74,147.98,136.34,134.21,133.90,133.59,133.29,129.97,127.48,127.25$, $121.92,119.73,118.32,117.55,116.36,115.37,113.59,105.11,19.07$. EIMS m/z: $\mathrm{M}^{+}$ 404.05. Anal. Calcd for $\mathrm{C}_{18} \mathrm{H}_{11} \mathrm{~F}_{3} \mathrm{~N}_{4} \mathrm{O}_{4}$ : C, 53.47; H, 2.74; $531 \mathrm{~N}, 13.86$. Found: $\mathrm{C}, 53.56 ; \mathrm{H}$, $2.77 ; \mathrm{N}, 13.78$.

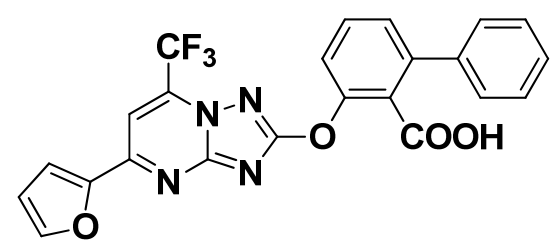

5-28

Data for 3-((5-(Furan-2-yl)-7-(trifluoromethyl)-[1,2,4]-triazolo[1,5-a]pyrimidin-2yl)oxy)-[1,1' -biphenyl]-2-carbox-ylic Acid (5-28). White solid, 30\% yield, mp: 228$229^{\circ} \mathrm{C} .{ }^{1} \mathrm{H}$ NMR $\left(600 \mathrm{MHz}, \mathrm{DMSO}-d_{6} \delta\right): 13.37(\mathrm{~s}, 1 \mathrm{H}), 8.15(\mathrm{~d}, J=6.0 \mathrm{~Hz}, 2 \mathrm{H}), 7.80(\mathrm{~s}$, 1H), $7.62(\mathrm{t}, J=7.8 \mathrm{~Hz}, 1 \mathrm{H}), 7.50(\mathrm{dd}, J=17.4,7.8 \mathrm{~Hz}, 5 \mathrm{H}), 7.42(\mathrm{~s}, 1 \mathrm{H}), 7.38(\mathrm{~d}, J=7.8$ $\mathrm{Hz}, 1 \mathrm{H}), 6.86(\mathrm{~s}, 1 \mathrm{H}) .{ }^{13} \mathrm{C}$ NMR (125 MHz, DMSO- $\left.d_{6} \delta\right): 169.84,167.31,154.57,151.54$, $149.99,149.74,148.04,140.12,139.10,134.24,133.94,133.63,133.32,130.11,128.51$, $128.17,127.85,127.14,126.77,119.73,117.55,116.45,113.63,105.22$. EIMS m/z: $\mathbf{M}^{+}$ 
355

356

357

358

359

360

361

362 363

364

365

366

367

368

369

370

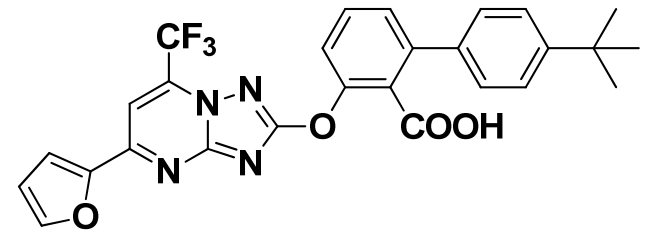

5-29

Data for 4' -(tert-Butyl)-3-((5-(furan-2-yl)-7-(trifluorometh-yl)-[1,2,4]triazolo[1,5a]pyrimidin-2-yl)oxy)-[1,1' -biphenyl]-2-carboxylic Acid (5-29). White solid, 57\% yield, mp: $225-226{ }^{\circ} \mathrm{C} .{ }^{1} \mathrm{H}$ NMR (600 MHz, DMSO- $d_{6} \delta$ ): $13.30(\mathrm{~s}, 1 \mathrm{H}), 8.10(\mathrm{~s}, 2 \mathrm{H}), 7.79(\mathrm{~d}, J$ $=3.0 \mathrm{~Hz}, 1 \mathrm{H}), 7.59(\mathrm{~s}, 1 \mathrm{H}), 7.49(\mathrm{~d}, J=7.8 \mathrm{~Hz}, 3 \mathrm{H}), 7.43(\mathrm{~d}, J=7.8 \mathrm{~Hz}, 2 \mathrm{H}), 7.36(\mathrm{~d}, J=$ $7.8 \mathrm{~Hz}, 1 \mathrm{H}), 6.86(\mathrm{~s}, 1 \mathrm{H}), 1.30(\mathrm{~d}, J=26.8 \mathrm{~Hz}, 9 \mathrm{H}) .{ }^{13} \mathrm{C}$ NMR $\left(125 \mathrm{MHz}, \mathrm{DMSO}-d_{6} \delta\right):$ $169.83,167.38,154.56,151.53,150.25,149.99,149.68,148.04,139.97,136.18,134.24$, $133.93,133.63,133.32,130.04,127.87,127.04,126.70,125.34,121.91,119.73,119.48$, 117.55, 116.45, 115.37, 113.63, 105.24, 34.29, 31.06. EIMS m/z: $\mathrm{M}^{+}$522.36. Anal. Calcd for $\mathrm{C}_{27} \mathrm{H}_{21} \mathrm{~F}_{3} \mathrm{~N}_{4} \mathrm{O}_{4}: \mathrm{C}, 62.07 ; \mathrm{H}, 4.05 ; \mathrm{N}, 10.72$. Found: C, 62.28; H, 4.04; N, 10.44 .

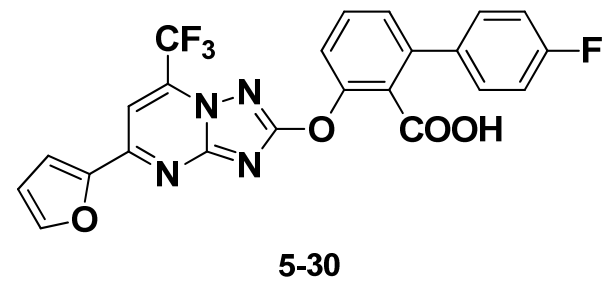

Data for 4 ' -Fluoro-3-((5-(furan-2-yl)-7-(trifluoromethyl)-[1,2,4]triazolo[1,5a]pyrimidin-2-yl)oxy)-[1,1' -biphenyl]-2-carboxylic Acid (5-30). White solid, 31\% yield, mp: $216-217{ }^{\circ} \mathrm{C} .{ }^{1} \mathrm{H}$ NMR (600 MHz, DMSO-d 6 $\left.\delta\right): 13.39(\mathrm{~s}, 1 \mathrm{H}), 8.13(\mathrm{~d}, J=3.0 \mathrm{~Hz}$, 2H), $7.79(\mathrm{~s}, 1 \mathrm{H}), 7.61(\mathrm{t}, J=7.8 \mathrm{~Hz}, 1 \mathrm{H}), 7.51(\mathrm{~d}, J=7.8 \mathrm{~Hz}, 3 \mathrm{H}), 7.37$ (d, $J=7.8 \mathrm{~Hz}, 1 \mathrm{H})$, $7.31(\mathrm{t}, J=8.4 \mathrm{~Hz}, 2 \mathrm{H}), 6.85(\mathrm{~s}, 1 \mathrm{H}) .{ }^{13} \mathrm{C}$ NMR (125 MHz, DMSO-d $\left.d_{6} \delta\right): 169.77,167.21$, 
371 372 373 374

375 376 377 378 379 380 381 382 383 384 Calcd for $\mathrm{C}_{24} \mathrm{H}_{14} \mathrm{~F}_{4} \mathrm{~N}_{4} \mathrm{O}_{4}$ : C, 57.84; H, 2.83; N, 11.24. Found: C, 57.98; H, 2.67; N, 11.48.

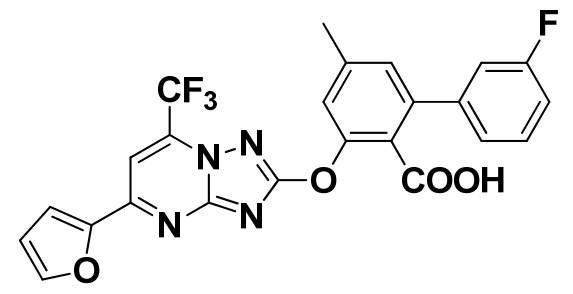

385

386

$162.93,160.98,154.56,151.55,149.98,149.72,148.05,139.05,135.44,133.94,133.63$, $130.29,130.22,130.16,127.11,126.77,121.89,119.83,119.72,117.54,116.47,115.48$ 115.31, 113.63, 105.24. EIMS m/z: $\mathrm{M}^{+}$484.08. Anal. Calcd for $\mathrm{C}_{23} \mathrm{H}_{12} \mathrm{~F}_{4} \mathrm{~N}_{4} \mathrm{O}_{4}: \mathrm{C}, 57.03 ; \mathrm{H}$, 2.50; N, 11.57. Found: C, 57.26; H, 2.76; N, 11.35.

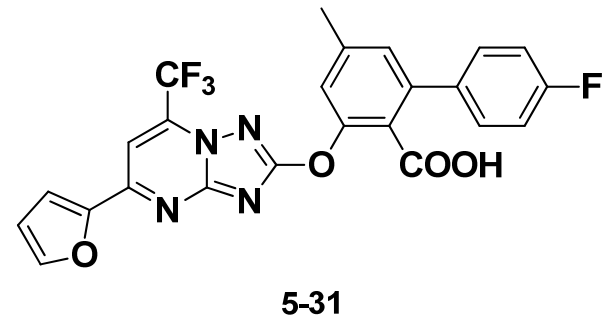

Data for 4 ' -Fluoro-3-((5-(furan-2-yl)-7-(trifluoromethyl)-[1,2,4]triazolo[1,5a]pyrimidin-2-yl)oxy)-5-methyl-[1,1' -bi-phenyl]-2-carboxylic Acid (5-31). White solid, 55\% yield, mp: $222-223{ }^{\circ} \mathrm{C} .{ }^{1} \mathrm{H}$ NMR (600 MHz, DMSO-d 6 $\delta$ ): $13.23(\mathrm{~s}, 1 \mathrm{H}), 8.14(\mathrm{~s}, 2 \mathrm{H})$, $7.80(\mathrm{~d}, J=3.6 \mathrm{~Hz}, 1 \mathrm{H}), 7.49(\mathrm{dd}, J=8.4,5.4 \mathrm{~Hz}, 2 \mathrm{H}), 7.31(\mathrm{dd}, J=10.2,7.8 \mathrm{~Hz}, 3 \mathrm{H}), 7.19$ $(\mathrm{s}, 1 \mathrm{H}), 6.86(\mathrm{~d}, J=1.8 \mathrm{~Hz}, 1 \mathrm{H}), 2.40(\mathrm{~s}, 3 \mathrm{H}) .{ }^{13} \mathrm{C}$ NMR $\left(125 \mathrm{MHz}, \mathrm{DMSO}-d_{6} \delta\right): 169.88$, $167.36,162.89,160.94,154.56,151.51,150.00,149.92,148.03,140.37,139.03,135.69$, $135.67,134.24,133.94,133.63,133.33,130.22,130.16,127.49,124.29,121.93,120.11$, 119.74, 117.56, 116.42, 115.40, 115.23, 113.63, 105.20, 20.80. EIMS m/z: $\mathrm{M}^{+}$498.00. Anal.

Data for 3 ' -Fluoro-3-((5-(furan-2-yl)-7-(trifluoromethyl)-[1,2,4]triazolo[1,5a]pyrimidin-2-yl)oxy)-5-methyl-[1,1' -bi-phenyl]-2-carboxylic Acid (5-32). White solid, 
388

389

390

391

392

393

394

395

396

397

398

399

400

401

402

403

404

405

406

36\% yield, mp: $215-216{ }^{\circ} \mathrm{C} .{ }^{1} \mathrm{H}$ NMR $\left(600 \mathrm{MHz}, \mathrm{DMSO}^{-d_{6}} \delta\right): 13.33(\mathrm{~s}, 1 \mathrm{H}), 8.15(\mathrm{~d}, J=$ $6.0 \mathrm{~Hz}, 2 \mathrm{H}), 7.81(\mathrm{~d}, J=3.0 \mathrm{~Hz}, 1 \mathrm{H}), 7.56^{-7.48}(\mathrm{~m}, 1 \mathrm{H}), 7.34(\mathrm{~s}, 1 \mathrm{H}), 7.33^{-7.22}(\mathrm{~m}, 4 \mathrm{H})$

$6.86(\mathrm{~s}, 1 \mathrm{H}), 2.41(\mathrm{~s}, 3 \mathrm{H}) .{ }^{13} \mathrm{C}$ NMR $\left(125 \mathrm{MHz}, \mathrm{DMSO}-d_{6} \delta\right): 169.85,167.26,162.92$, $160.99,154.56,151.53,149.99,149.94,148.04,141.64,141.57,140.54,138.64,134.24$, $133.94,133.64,133.33,130.51,130.45,127.47,124.42,124.40,124.24,121.92,120.54$, $119.74,117.56,116.44,115.38,115.04,114.87,114.69,114.52,113.63,105.20,20.80$

EIMS m/z: $\mathrm{M}^{+}$498.07. Anal. Calcd for $\mathrm{C}_{24} \mathrm{H}_{14} \mathrm{~F}_{4} \mathrm{~N}_{4} \mathrm{O}_{4}$ : C, 57.84; H, 2.83; N, 11.24. Found: C, 57.80; H, 3.00; N, 11.34 .

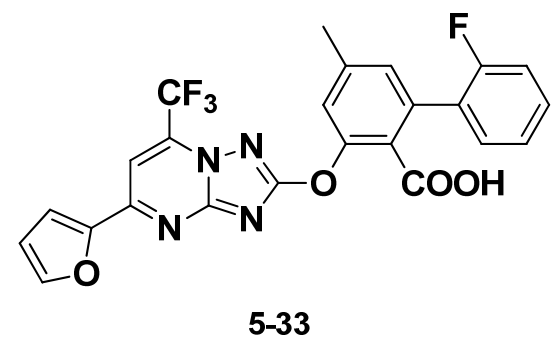

Data for 2 ' -Fluoro-3-((5-(furan-2-yl)-7-(trifluoromethyl)-[1,2,4]triazolo[1,5a]pyrimidin-2-yl)oxy)-5-methyl-[1,1' -bi-phenyl]-2-carboxylic Acid (5-33). White solid, 44\% yield, mp: $220-221{ }^{\circ} \mathrm{C} .{ }^{1} \mathrm{H}$ NMR (600 MHz, DMSO-d 6 $\delta$ ): 13.01 (s, 1H), $8.14(\mathrm{~s}, 2 \mathrm{H})$, $7.79(\mathrm{~d}, J=3.6 \mathrm{~Hz}, 1 \mathrm{H}), 7.45(\mathrm{dd}, J=13.2,6.0 \mathrm{~Hz}, 1 \mathrm{H}), 7.40(\mathrm{t}, J=7.8 \mathrm{~Hz}, 1 \mathrm{H}), 7.35(\mathrm{~s}$, 1H), $7.29(\mathrm{dd}, J=13.2,6.6 \mathrm{~Hz}, 2 \mathrm{H}), 7.18(\mathrm{~s}, 1 \mathrm{H}), 6.86(\mathrm{dd}, J=3.6,1.8 \mathrm{~Hz}, 1 \mathrm{H}), 2.40(\mathrm{~s}$, 3H). ${ }^{13} \mathrm{C}$ NMR (125 MHz, DMSO-d 6 $\left.\delta\right): 169.98,166.52,159.89,157.94,154.53,151.47$, $150.26,150.00,148.03,140.74,134.84,134.21,133.91,133.60,133.30,131.03,131.01$, $130.03,129.97,128.52,127.09,126.97,124.58,124.33,124.31,121.92,121.15,119.73$, 117.55, 116.39, 115.51, 115.33, 113.63, 105.14, 20.73. EIMS m/z: $\mathrm{M}^{+}$498.06. Anal. Calcd for $\mathrm{C}_{24} \mathrm{H}_{14} \mathrm{~F}_{4} \mathrm{~N}_{4} \mathrm{O}_{4}$ : C, 57.84; H, 2.83; N, 11.24. Found: C, 57.70; H, 2.68; N, 11.46. 
407

408

409

410

411

412

413

414

415

416

417

418

419

420

421

422

423

424

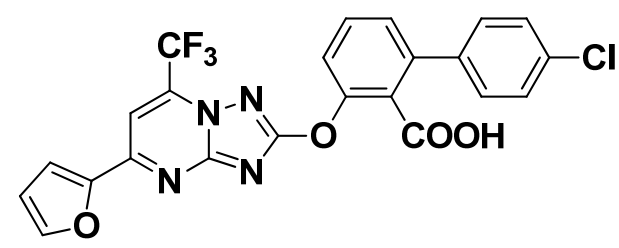

5-34

Data for 4 ' -Chloro-3-((5-(furan-2-yl)-7-(trifluoromethyl)-[1,2,4]triazolo[1,5a]pyrimidin-2-yl)oxy)-[1,1' -biphenyl]-2-carboxylic Acid (5-34). White solid, 33\% yield, mp: $220-221{ }^{\circ} \mathrm{C} .{ }^{1} \mathrm{H}$ NMR (600 MHz, DMSO-d 6 $\left.\delta\right): 13.36(\mathrm{~s}, 1 \mathrm{H}), 8.14(\mathrm{~d}, J=7.2 \mathrm{~Hz}$, 2H), $7.80(\mathrm{~d}, J=3.6 \mathrm{~Hz}, 1 \mathrm{H}), 7.63(\mathrm{t}, J=7.8 \mathrm{~Hz}, 1 \mathrm{H}), 7.54(\mathrm{dd}, J=13.2,8.4 \mathrm{~Hz}, 2 \mathrm{H}), 7.50$ $(\mathrm{d}, J=8.4 \mathrm{~Hz}, 1 \mathrm{H}), 7.39(\mathrm{~d}, J=7.8 \mathrm{~Hz}, 1 \mathrm{H}), 7.33(\mathrm{t}, J=8.4 \mathrm{~Hz}, 2 \mathrm{H}), 6.86(\mathrm{~s}, 1 \mathrm{H}) .{ }^{13} \mathrm{C}$ NMR (125 MHz, DMSO- $\left.d_{6} \delta\right): 169.78,167.11,164.73,154.56,151.55,149.99,149.76$, $148.07,138.75,137.80,133.93,132.85,132.85,130.02,129.94,128.52,127.42,126.67$ 124.67, 122.54, 120.08, 120.03, 119.16, 116.48, 113.65, 105.29. EIMS m/z: $\mathrm{M}^{+} 500.13$. Anal. Calcd for $\mathrm{C}_{23} \mathrm{H}_{12} \mathrm{ClF}_{3} \mathrm{~N}_{4} \mathrm{O}_{4}$ : C, 55.16; H, 624 2.42; N, 11.19. Found: C, 55.23; H, $2.45 ; \mathrm{N}, 11.02$.

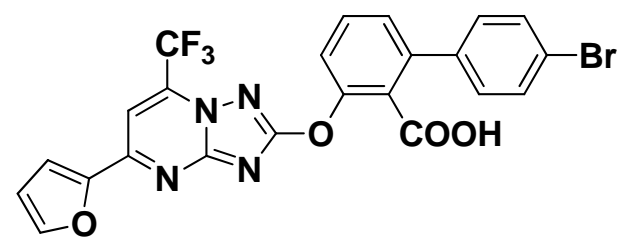

5-35

Data for 4 ' -Bromo-3-((5-(furan-2-yl)-7-(trifluoromethyl)-[1,2,4]triazolo[1,5a]pyrimidin-2-yl)oxy)-[1,1' -biphenyl]-2-carboxylic Acid (5-35). White solid, 31\% yield, mp: $219-220{ }^{\circ} \mathrm{C} .{ }^{1} \mathrm{H}$ NMR (600 MHz, DMSO-d 6 $\left.\delta\right): 13.42(\mathrm{~s}, 1 \mathrm{H}), 8.15-8.11(\mathrm{~m}, 2 \mathrm{H})$, $7.79(\mathrm{~d}, J=3.6 \mathrm{~Hz}, 1 \mathrm{H}), 7.68(\mathrm{~d}, J=8.4 \mathrm{~Hz}, 2 \mathrm{H}), 7.63(\mathrm{t}, J=7.8 \mathrm{~Hz}, 1 \mathrm{H}), 7.53(\mathrm{~d}, J=8.4$ $\mathrm{Hz}, 1 \mathrm{H}), 7.43(\mathrm{~d}, J=8.4 \mathrm{~Hz}, 2 \mathrm{H}), 7.38(\mathrm{~d}, J=7.8 \mathrm{~Hz}, 1 \mathrm{H}), 6.86(\mathrm{dd}, J=3.6,1.8 \mathrm{~Hz}, 1 \mathrm{H})$.

${ }^{13} \mathrm{C}$ NMR (125 MHz, DMSO- $d_{6} \delta$ ): 169.75, 167.09, 154.55, 151.55, 149.97, 149.80, 
429

430 431 432 433 434 435 436 437 438 439

440

441 442

$148.04,138.93,138.28,134.24,133.94,133.63,133.33,131.46,130.33,130.30,126.92$, 126.64, 121.51, 120.13, 119.71, 117.53, 116.47, 113.62, 105.24. EIMS m/z: $\mathrm{M}^{+} 545.81$. Anal. Calcd for $\mathrm{C}_{23} \mathrm{H}_{12} \mathrm{BrF}_{3} \mathrm{~N}_{4} \mathrm{O}_{4}: \mathrm{C}, 50.66 ; \mathrm{H}, 2.22 ; \mathrm{N}, 10.28$. Found: $\mathrm{C}, 50.52 ; \mathrm{H}, 2.30 ; \mathrm{N}$, 10.02

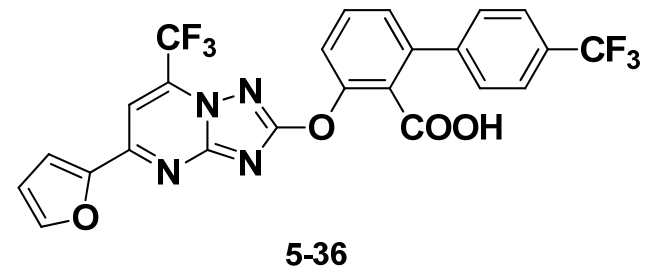

Data for 3-((5-(Furan-2-yl)-7-(trifluoromethyl)-[1,2,4]-triazolo[1,5-a]pyrimidin-2yl)oxy)-4' -(trifluoromethyl)-[1,1' -biphenyl]-2-carboxylic Acid (5-36). White solid, 27\% yield, mp: $216-217^{\circ} \mathrm{C} .{ }^{1} \mathrm{H}$ NMR $\left(600 \mathrm{MHz}, \mathrm{DMSO}-d_{6} \delta\right): 13.46(\mathrm{~s}, 1 \mathrm{H}), 8.14(\mathrm{~d}, J=4.8$ $\mathrm{Hz}, 2 \mathrm{H}), 7.86(\mathrm{~d}, J=7.8 \mathrm{~Hz}, 2 \mathrm{H}), 7.80(\mathrm{~d}, J=3.6 \mathrm{~Hz}, 1 \mathrm{H}), 7.70(\mathrm{~d}, J=7.8 \mathrm{~Hz}, 2 \mathrm{H}), 7.67$ (s, 1H), $7.58(\mathrm{~d}, J=8.4 \mathrm{~Hz}, 1 \mathrm{H}), 7.43(\mathrm{~d}, J=7.8 \mathrm{~Hz}, 1 \mathrm{H}), 6.86(\mathrm{~d}, J=1.8 \mathrm{~Hz}, 1 \mathrm{H}), 2.09$ (s, 3H). ${ }^{13} \mathrm{C}$ NMR (125 MHz, DMSO- $d_{6} \delta$ ): 169.77, 166.97, 154.57, 151.74, 151.57, 150.00, $149.81,148.08,147.70,143.31,143.03,138.86,138.70,133.95,133.65,130.26,129.11$, $128.45,128.20,126.80,125.41,125.38,125.30,123.14,120.61,119.73,117.55,116.50$, 113.66, 105.30. EIMS m/z: $\mathrm{M}^{+}$534.11. Anal. Calcd for $\mathrm{C}_{24} \mathrm{H}_{12} \mathrm{~F}_{6} \mathrm{~N}_{4} \mathrm{O}_{4}$ : C, 53.94; H, 2.26; N, 10.48. Found: C, 53.89; H, 2.35; N, 10.24.

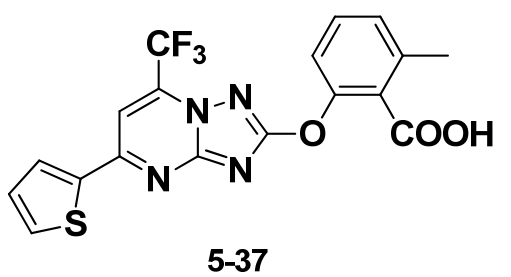

Data for 2-Methyl-6-((5-(thiophen-2-yl)-7-(trifluorometh-yl)-[1,2,4]triazolo[1,5a]pyrimidin-2-yl)oxy)benzoic Acid (5-37). Yellow solid, 55\% yield, mp: $234-235{ }^{\circ} \mathrm{C} .{ }^{1} \mathrm{H}$ 
443 NMR (600 MHz, DMSO-d $\left.d_{6} \delta\right): 13.36(\mathrm{~s}, 1 \mathrm{H}), 8.43(\mathrm{~s}, 2 \mathrm{H}), 7.99$ (d, J = $\left.4.8 \mathrm{~Hz}, 1 \mathrm{H}\right), 7.43$ $444(\mathrm{t}, J=7.8 \mathrm{~Hz}, 1 \mathrm{H}), 7.32(\mathrm{~d}, J=4.2 \mathrm{~Hz}, 1 \mathrm{H}), 7.28(\mathrm{~d}, J=8.4 \mathrm{~Hz}, 1 \mathrm{H}), 7.23(\mathrm{~d}, J=7.8 \mathrm{~Hz}$, $4451 \mathrm{H}), 2.37(\mathrm{~s}, 3 \mathrm{H}) .{ }^{13} \mathrm{C}$ NMR (125 MHz, DMSO-d $\left.d_{6} \delta\right): 169.89,167.35,156.11,154.25$, $446149.75,141.05,136.32,134.06,133.86,133.76,133.45,133.15,132.05,129.99,129.31$, $447127.49,127.25,122.01,119.83,118.39,117.65,115.46,105.49,19.07$. EIMS m/z: $\mathrm{M}^{+}$ 448 420.55. Anal. Calcd for $\mathrm{C}_{18} \mathrm{H}_{11} \mathrm{~F}_{3} \mathrm{~N}_{4} \mathrm{O}_{3} \mathrm{~S}: \mathrm{C}, 51.43 ; \mathrm{H}, 2.64 ; \mathrm{N}, 13.33$. Found: C, 51.51; H, $449 \quad 2.53 ; \mathrm{N}, 13.58$.

450

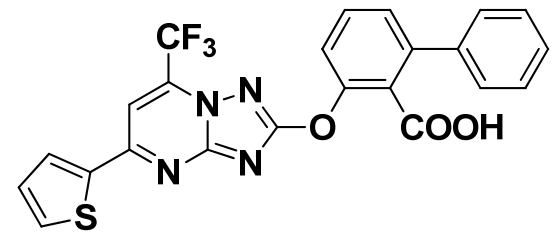

5-38

451 Data for 3-((5-(Thiophen-2-yl)-7-(trifluoromethyl)-[1,2,4]-triazolo[1,5-a]pyrimidin-2452 yl)oxy)-[1,1' -biphenyl]-2-carbox-ylic Acid (5-38). White solid, 27\% yield, mp: 246$453247{ }^{\circ} \mathrm{C} .{ }^{1} \mathrm{H}$ NMR (600 MHz, DMSO-d 6 ) $13.33(\mathrm{~s}, 1 \mathrm{H}), 8.43$ (s, 2H), 8.00 (d, J = 4.8 Hz, $4541 \mathrm{H}), 7.62(\mathrm{t}, J=7.8 \mathrm{~Hz}, 1 \mathrm{H}), 7.53-7.45(\mathrm{~m}, 5 \mathrm{H}), 7.42(\mathrm{t}, J=6.6 \mathrm{~Hz}, 1 \mathrm{H}), 7.38(\mathrm{~d}, J=7.8$ $455 \mathrm{~Hz}, 1 \mathrm{H}), 7.32(\mathrm{t}, J=4.2 \mathrm{~Hz}, 1 \mathrm{H}) .{ }^{13} \mathrm{C}$ NMR (125 MHz, DMSO-d 6 $\left.\delta\right): 169.76,167.28$, $456156.18,154.26,149.74,141.05,140.10,139.09,133.94,133.80,133.50,133.19,132.11$, $457130.12,129.35,128.50,128.16,127.84,127.16,126.77,119.83,117.64,105.59,99.47$. 458 EIMS m/z: $\mathrm{M}^{+}$482.56. Anal. Calcd for $\mathrm{C}_{23} \mathrm{H}_{13} \mathrm{~F}_{3} \mathrm{~N}_{4} \mathrm{O}_{3} \mathrm{~S}: \mathrm{C}, 57.26 ; \mathrm{H}, 2.72 ; \mathrm{N}, 11.61$. Found: 459 C, 57.24; H, 2.65; N, 11.73 .

460

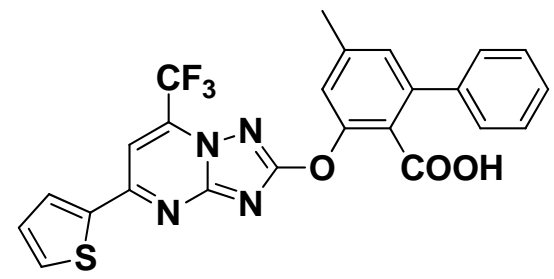


462 a]pyrimidin-2-yl)oxy)-[1,1' -biphenyl]-2-carboxylic Acid (5-39). Yellow solid, 49\% yield, 463 mp: $220-221{ }^{\circ} \mathrm{C} .{ }^{1} \mathrm{H}$ NMR (600 MHz, DMSO-d $) \quad \delta \quad 13.16$ (s, 1H), 8.43 (s, 2H), 7.99 (d, $J$ $464=4.8 \mathrm{~Hz}, 1 \mathrm{H}), 7.46(\mathrm{~d}, J=3.6 \mathrm{~Hz}, 4 \mathrm{H}), 7.41(\mathrm{~s}, 1 \mathrm{H}), 7.35-7.31(\mathrm{~m}, 1 \mathrm{H}), 7.30(\mathrm{~s}, 1 \mathrm{H}), 7.20$ 465 (s, 1H), $2.41(\mathrm{~s}, 3 \mathrm{H}) .{ }^{13} \mathrm{C}$ NMR $\left(125 \mathrm{MHz}, \mathrm{DMSO}_{-} \mathrm{d}_{6} \delta\right): 169.93,167.50,156.18,154.30$, $466149.96,141.07,140.37,140.14,139.36,134.13,133.93,133.83,133.53,133.22,132.11$, 467 $129.36,128.48,128.16,127.78,127.56,124.41,122.07,120.18,119.88,117.70,115.53$, 468 105.57, 20.86. EIMS m/z: $\mathrm{M}^{+}$496.88. Anal. Calcd for $\mathrm{C}_{24} \mathrm{H}_{15} \mathrm{~F}_{3} \mathrm{~N}_{4} \mathrm{O}_{3} \mathrm{~S}: \mathrm{C}, 58.06 ; \mathrm{H}, 3.05 ; \mathrm{N}$, 469 11.29. Found: C, 57.99; H, 3.03; N, 11.27.

470

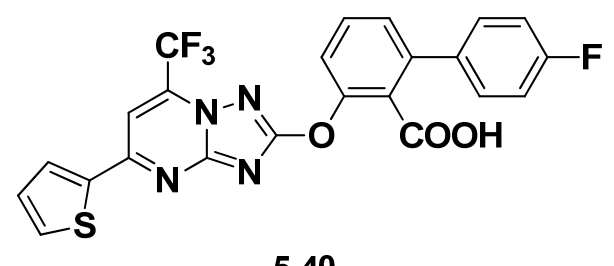

471

Data for 4' -Fluoro-3-((5-(thiophen-2-yl)-7-(trifluorometh-yl)-[1,2,4]triazolo[1,5472 a]pyrimidin-2-yl)oxy)-[1,1' -biphenyl]-2-carboxylic Acid (5-40). White solid, 18\% yield, 473 mp: $226-227^{\circ} \mathrm{C} .{ }^{1} \mathrm{H}$ NMR $\left(600 \mathrm{MHz}, \mathrm{DMSO}^{-d_{6}} \delta\right.$ ): 13.39 (s, 1H), 8.43 (s, 2H), 8.00 (d, J $474=3.6 \mathrm{~Hz}, 1 \mathrm{H}), 7.62(\mathrm{t}, J=7.8 \mathrm{~Hz}, 1 \mathrm{H}), 7.56-7.48(\mathrm{~m}, 3 \mathrm{H}), 7.38(\mathrm{~d}, J=7.8 \mathrm{~Hz}, 1 \mathrm{H}), 7.33(\mathrm{~d}$, $475 J=8.4 \mathrm{~Hz}, 3 \mathrm{H}) .{ }^{13} \mathrm{C}$ NMR $\left(125 \mathrm{MHz}, \mathrm{DMSO}-d_{6} \delta\right): 169.75,167.24,162.95,161.00$, $476156.22,154.28,149.77,141.05,139.09,135.48,135.46,134.12,133.95,133.83,133.52$, $477133.22,132.13,130.30,130.23,129.36,127.14,126.81,122.03,119.98,119.84,117.66$, 478 115.49, 115.32, 105.61. EIMS m/z: $\mathrm{M}^{+}$500.88. Anal. Calcd for $\mathrm{C}_{23} \mathrm{H}_{12} \mathrm{~F}_{4} \mathrm{~N}_{4} \mathrm{O}_{3} \mathrm{~S}: \mathrm{C}$, 55.20; $479 \quad$ H, 2.42; N, 11.20. Found: C, 55.46; H, 2.39; N, 11.33. 


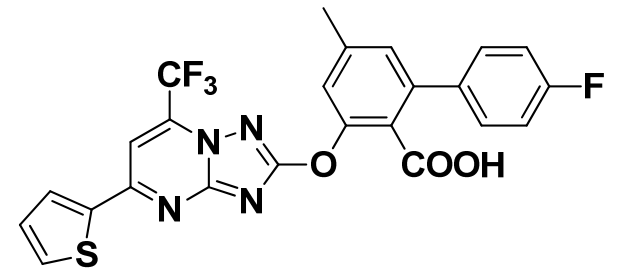

480

$5-41$

481

Data for 4 ' -Fluoro-5-methyl-3-((5-(thiophen-2-yl)-7-(tri-fluoromethyl)482 [1,2,4]triazolo[1,5-a]pyrimidin-2-yl)oxy)-[1,1' -biphenyl]-2-carboxylic Acid (5-41). White 483 solid, 65\% yield, mp: $222-223{ }^{\circ} \mathrm{C} .{ }^{1} \mathrm{H}$ NMR (600 MHz, DMSO-d ${ }_{6} \delta$ ): $13.22(\mathrm{~s}, 1 \mathrm{H}), 8.43$ $484(\mathrm{~s}, 2 \mathrm{H}), 8.00(\mathrm{~d}, J=4.8 \mathrm{~Hz}, 1 \mathrm{H}), 7.54-7.46(\mathrm{~m}, 2 \mathrm{H}), 7.31(\mathrm{t}, J=9.0 \mathrm{~Hz}, 4 \mathrm{H}), 7.20(\mathrm{~s}, 1 \mathrm{H})$, 485 $2.40(\mathrm{~s}, 3 \mathrm{H}) .{ }^{13} \mathrm{C}$ NMR $\left(125 \mathrm{MHz}, \mathrm{DMSO}_{-} \delta\right.$ ): 169.85, 167.38, 162.89, 160.94, 156.16, 486 $154.27,149.94,141.05,140.40,139.04,135.71,135.69,134.11,133.92,133.81,133.51$ 487 $133.20,132.10,130.23,130.16,129.33,127.53,124.38,122.04,120.26,119.85,117.67$ 488 115.40, 115.23, 105.56, 20.81. EIMS m/z: $\mathrm{M}^{+}$514.00. Anal. Calcd for $\mathrm{C}_{24} \mathrm{H}_{14} \mathrm{~F}_{4} \mathrm{~N}_{4} \mathrm{O}_{3} \mathrm{~S}: \mathrm{C}$, 489 56.03; H, 2.74; N, 10.89. Found: C, 56.00; H, 2.72; N, 11.02.

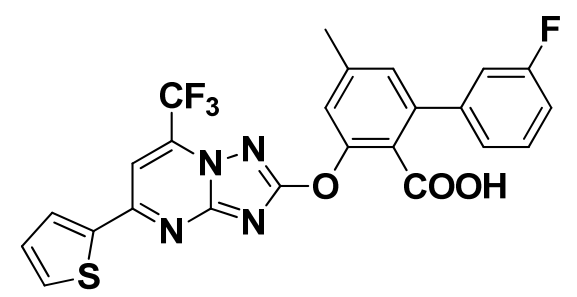

490 $5-42$

491

Data for 3 ' -Fluoro-5-methyl-3-((5-(thiophen-2-yl)-7-(tri-fluoromethyl)492 [1,2,4]triazolo[1,5-a]pyrimidin-2-yl)oxy)-[1,1 ' -biphenyl]-2-carboxylic Acid (5-42). 493 Yellow solid, 59\% yield, mp: $220-221{ }^{\circ} \mathrm{C} .{ }^{1} \mathrm{H}$ NMR $\left(600 \mathrm{MHz}, \mathrm{DMSO}-d_{6} \delta\right)$ : 13.29 (s, $4941 \mathrm{H}), 8.43(\mathrm{~s}, 2 \mathrm{H}), 8.00(\mathrm{~d}, J=4.8 \mathrm{~Hz}, 1 \mathrm{H}), 7.51(\mathrm{~d}, J=6.6 \mathrm{~Hz}, 1 \mathrm{H}), 7.36^{-7.22}(\mathrm{~m}, 6 \mathrm{H})$, $4952.41(\mathrm{~s}, 3 \mathrm{H}) .{ }^{13} \mathrm{C}$ NMR (125 MHz, DMSO-d $\left.d_{6} \delta\right): 169.82,167.27,162.93,160.99,156.18$, $496154.27,149.96,141.65,141.59,141.04,140.58,138.67,134.12,133.93,133.82,133.51$, 
501

502

503

504

505

506

507

508

509

510

511

512

513

514

$133.21,132.11,130.51,130.45,129.33,127.50,124.42,124.40,124.32,122.04,120.69$, $119.85,117.67,115.48,115.04,114.87,114.68,114.52,105.61,20.80$. EIMS m/z: $\mathrm{M}^{+}$ 514.23. Anal. Calcd for $\mathrm{C}_{24} \mathrm{H}_{14} \mathrm{~F}_{4} \mathrm{~N}_{4} \mathrm{O}_{3} \mathrm{~S}$ : C, 56.03; H, 2.74; N, 10.89. Found: C, 55.96; H, $2.57 ; \mathrm{N}, 11.01$.

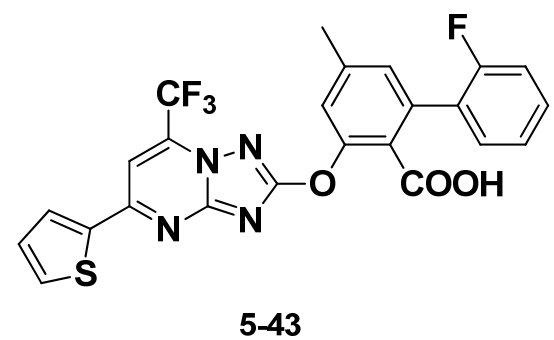

Data for 2 ' -Fluoro-5-methyl-3-((5-(thiophen-2-yl)-7-(tri-fluoromethyl)[1,2,4]triazolo[1,5-a]pyrimidin-2-yl)oxy)-[1,1 ' -biphenyl]-2-carboxylic Acid (5-43). Yellow solid, 41\% yield, mp: $217-218{ }^{\circ} \mathrm{C} .{ }^{1} \mathrm{H}$ NMR $\left(600 \mathrm{MHz}, \mathrm{DMSO}-\mathrm{d}_{6} \delta\right): 13.01$ (s, 1H), $8.43(\mathrm{~s}, 2 \mathrm{H}), 7.99(\mathrm{~s}, 1 \mathrm{H}), 7.42(\mathrm{~d}, J=27.6 \mathrm{~Hz}, 3 \mathrm{H}), 7.38-7.23(\mathrm{~m}, 3 \mathrm{H}), 7.18(\mathrm{~s}, 1 \mathrm{H})$, 2.41 (s, 3H). ${ }^{13} \mathrm{C}$ NMR (125 MHz, DMSO-d $\left.6 \quad \delta\right): 169.90,166.50,159.87,157.92,156.11$, $154.23,150.25,141.06,140.75,134.82,133.93,133.77,133.46,132.10,131.02,131.00$, $130.03,129.96,129.35,128.53,127.08,126.95,124.61,124.33,124.31,122.02,121.24$, 119.83, 117.65, 115.50, 115.33, 105.55, 105.53, 20.72. EIMS m/z: $\mathrm{M}^{+}$514.43. Anal. Calcd for $\mathrm{C}_{24} \mathrm{H}_{14} \mathrm{~F}_{4} \mathrm{~N}_{4} \mathrm{O}_{3} \mathrm{~S}: \mathrm{C}, 56.03 ; \mathrm{H}, 2.74 ; \mathrm{N}, 10.89$. Found: C, 56.03; H, 2.58; N, 11.18 .

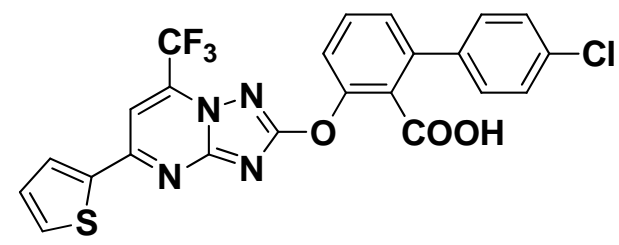

$5-44$

Data for 4' -Chloro-3-((5-(thiophen-2-yl)-7-(trifluorometh-yl)-[1,2,4]triazolo[1,5a]pyrimidin-2-yl)oxy)-[1,1' -biphenyl]-2-carboxylic Acid (5-44). Yellow solid, 65\% yield, mp: $210-211^{\circ} \mathrm{C} .{ }^{1} \mathrm{H}$ NMR (600 MHz, DMSO-d 6 ) $): 13.42$ (s, 1H), 8.43 (s, 2H), 8.00 (d, J 
515

516 517 518 519 520

521

522

523

524

525

526

527

528

529

530

531

$=4.2 \mathrm{~Hz}, 1 \mathrm{H}), 7.62(\mathrm{~d}, J=7.8 \mathrm{~Hz}, 1 \mathrm{H}), 7.54(\mathrm{t}, J=11.4 \mathrm{~Hz}, 3 \mathrm{H}), 7.49(\mathrm{~d}, J=7.2 \mathrm{~Hz}, 2 \mathrm{H})$, $7.39(\mathrm{~d}, J=7.2 \mathrm{~Hz}, 1 \mathrm{H}), 7.33(\mathrm{~s}, 1 \mathrm{H}) .{ }^{13} \mathrm{C}$ NMR (125 MHz, DMSO-d 6 ) : 169.80, 167.20, $156.23,154.28,149.85,141.03,138.94,137.95,134.11,133.93,133.84,133.53,133.23$, $132.95,132.12,130.36,130.02,129.33,128.55,127.11,126.77,122.03,120.32,119.85$, 117.67, 115.48, 105.61. EIMS m/z: $\mathrm{M}^{+}$516.04. Anal. Calcd for $\mathrm{C}_{23} \mathrm{H}_{12} \mathrm{ClF}_{3} \mathrm{~N}_{4} \mathrm{O}_{3} \mathrm{~S}$ : $\mathrm{C}$, 53.44; H, 2.34; N, 10.84. Found: C, 53.28; H, 2.26; N, 11.04.

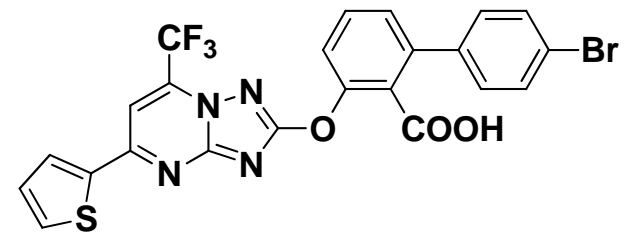

$5-45$

Data for 4' -Bromo-3-((5-(thiophen-2-yl)-7-(trifluorometh-yl)-[1,2,4]triazolo[1,5a]pyrimidin-2-yl)oxy)-[1,1' -biphenyl]-2-carboxylic Acid (5-45). Yellow solid, 49\% yield, mp: $217-218^{\circ} \mathrm{C} .{ }^{1} \mathrm{H}$ NMR (600 MHz, DMSO-d 6 ) $) 13.43(\mathrm{~s}, 1 \mathrm{H}), 8.43$ (s, 2H), 7.99 (s, 1H), $7.68(\mathrm{~d}, J=7.8 \mathrm{~Hz}, 2 \mathrm{H}), 7.62(\mathrm{~d}, J=7.8 \mathrm{~Hz}, 1 \mathrm{H}), 7.53(\mathrm{~d}, J=7.8 \mathrm{~Hz}, 1 \mathrm{H}), 7.43(\mathrm{~d}, J=$ $7.8 \mathrm{~Hz}, 2 \mathrm{H}), 7.38(\mathrm{~d}, J=7.8 \mathrm{~Hz}, 1 \mathrm{H}), 7.32(\mathrm{~s}, 1 \mathrm{H}) .{ }^{13} \mathrm{C}$ NMR $(125 \mathrm{MHz}$, DMSO-d $6 \quad \delta):$ $169.73,167.12,156.21,154.26,149.83,141.04,138.93,138.30,134.12,133.95,133.82$, $133.51,133.21,132.13,131.46,130.35,130.31,129.36,127.01,126.67,122.00,121.51$, 120.27, 119.83, 117.64, 115.46, 105.62. EIMS m/z: $\mathrm{M}^{+}$560.98. Anal. Calcd for $\mathrm{C}_{23} \mathrm{H}_{12} \mathrm{BrF}_{3} \mathrm{~N}_{4} \mathrm{O}_{3} \mathrm{~S}: \mathrm{C}, 49.21 ; \mathrm{H}, 2.15 ; \mathrm{N}, 9.98$. Found: C, 49.02; H, 2.10; N, 9.92.

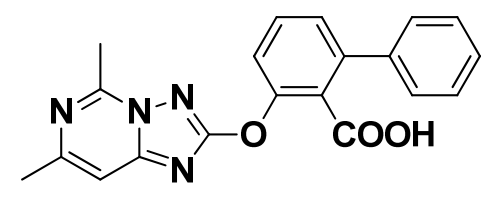

$5-46$

Data for 3-((5,7-Dimethyl-[1,2,4]triazolo[1,5-c]pyrimidin-2-yl)oxy)-[1,1' 2-carboxylic Acid (5-46). White solid, 45\% yield, mp: $215-216{ }^{\circ} \mathrm{C} .{ }^{1} \mathrm{H}$ NMR $(600 \mathrm{MHz}$, 
534

535

536

537 538

539

540

541

542

543

544

545

546

547

548

549

550

551

DMSO-d $\left._{6} \delta\right): 13.26(\mathrm{~s}, 1 \mathrm{H}), 7.58(\mathrm{t}, J=7.8 \mathrm{~Hz}, 1 \mathrm{H}), 7.47$ (d, $\left.J=4.8 \mathrm{~Hz}, 6 \mathrm{H}\right), 7.42(\mathrm{~d}, J=$ $3.0 \mathrm{~Hz}, 1 \mathrm{H}), 7.34(\mathrm{~d}, J=7.8 \mathrm{~Hz}, 1 \mathrm{H}), 2.77(\mathrm{~s}, 3 \mathrm{H}), 2.48(\mathrm{~s}, 3 \mathrm{H}) .{ }^{13} \mathrm{C} \mathrm{NMR}(125 \mathrm{MHz}$, $\left.\mathrm{DMSO}_{6} \delta\right): 168.68,167.35,154.96,152.10,149.98,149.06,140.07,139.13,130.11$, $128.48,128.16,127.81,127.16,126.58,119.96,105.75,23.43,19.25$. HRMS (MALDI): $[\mathrm{M}+\mathrm{Na}]^{+}$calcd for $\mathrm{C}_{20} \mathrm{H}_{16} \mathrm{~N}_{4} \mathrm{O}_{3}, 383.1120$; found, 383.1117 .

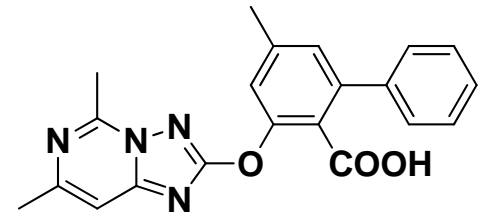

$5-47$

Data for 3-((5,7-Dimethyl-[1,2,4]triazolo[1,5-c]pyrimidin-2-yl)oxy)-5-methyl-[1,1' biphenyl]-2-carboxylic Acid (5-47). White solid, 45\% yield, mp: $211-212{ }^{\circ} \mathrm{C} .{ }^{1} \mathrm{H}$ NMR (400 MHz, DMSO- $\left.d_{6} \delta\right): 13.05(\mathrm{~s}, 1 \mathrm{H}), 7.51-7.42(\mathrm{~m}, 5 \mathrm{H}), 7.40(\mathrm{~s}, 1 \mathrm{H}), 7.24(\mathrm{~s}, 1 \mathrm{H})$, $7.16(\mathrm{~s}, 1 \mathrm{H}), 3.32(\mathrm{~s}, 3 \mathrm{H}), 2.78(\mathrm{~s}, 3 \mathrm{H}), 2.38(\mathrm{~s}, 3 \mathrm{H}) .{ }^{13} \mathrm{C}$ NMR $(125 \mathrm{MHz}$, DMSO-d 6 $\delta):$ $168.78,167.49,154.90,152.09,150.16,149.02,140.26,140.04,139.38,128.41,128.12$, $127.69,127.35,124.40,120.22,105.75,23.43,20.79,19.26$. HRMS (MALDI): $[\mathrm{M}+\mathrm{Na}]^{+}$ calcd for $\mathrm{C}_{21} \mathrm{H}_{18} \mathrm{~N}_{4} \mathrm{O}_{3}, 397.1277$; found, 397.1277.

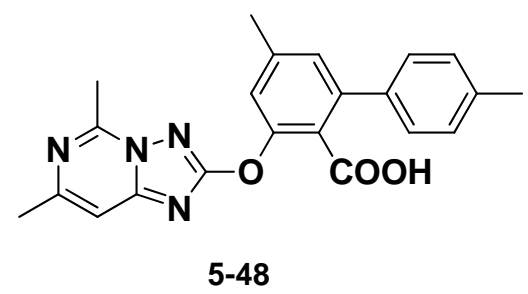

Data for 3-((5,7-Dimethyl-[1,2,4]triazolo[1,5-c]pyrimidin-2-yl)oxy)-4' ,5-dimethyl[1,1' -biphenyl]-2-carboxylic Acid (5-48). White solid, 33\% yield, mp: $214-215{ }^{\circ} \mathrm{C} .{ }^{1} \mathrm{H}$ NMR (600 M Hz, DMSO-d $d_{6} \delta$ ): $13.04(\mathrm{~s}, 1 \mathrm{H}), 7.46$ (s, 1H), 7.34 (d, J=7.2 Hz, 2H), 7.25 (d, $J=7.2 \mathrm{~Hz}, 2 \mathrm{H}), 7.21(\mathrm{~s}, 1 \mathrm{H}), 7.13(\mathrm{~s}, 1 \mathrm{H}), 2.77$ (s, 3H), 2.47 (s, 3H), 2.37 (s, 3H), 2.34 

$149.01,140.15,139.98,137.05,136.49,129.01,128.01,127.23,124.38,119.99,105.74$, 23.43, 20.81, 20.68, 19.25. HRMS (MALDI): $[\mathrm{M}+\mathrm{H}]^{+}$calcd for $\mathrm{C}_{22} \mathrm{H}_{20} \mathrm{~N}_{4} \mathrm{O}_{3}, 389.1614$; 555 found, 389.1610.

556

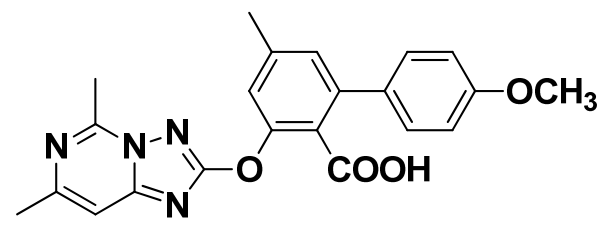

5-49

558

564 found, 405.1562.

565

566 nitro-[1,1' -biphenyl]-2-carboxylic Acid (5-50). White solid, $28 \%$ yield, mp: $215-216{ }^{\circ} \mathrm{C}$.

$568{ }^{1} \mathrm{H}$ NMR $\left(400 \mathrm{M} \mathrm{Hz}, \mathrm{DMSO}^{-} d_{6} \delta\right): 13.31(\mathrm{~s}, 1 \mathrm{H}), 8.32(\mathrm{~d}, J=8.0 \mathrm{~Hz}, 2 \mathrm{H}), 7.71(\mathrm{~d}, J=8.0$ 569

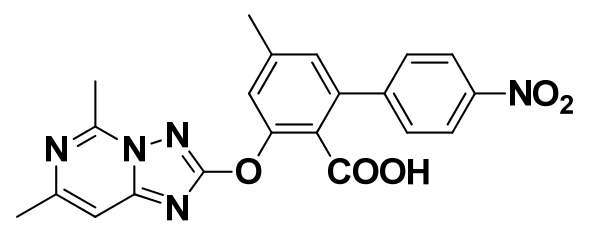

$5-50$
Data for 3-((5,7-Dimethyl-[1,2,4]triazolo[1,5-c]pyrimidin-2-yl)oxy)-5-methyl-4 ' $\mathrm{Hz}, 2 \mathrm{H}), 7.47(\mathrm{~s}, 1 \mathrm{H}), 7.35(\mathrm{~s}, 1 \mathrm{H}), 7.25(\mathrm{~s}, 1 \mathrm{H}), 2.77(\mathrm{~s}, 3 \mathrm{H}), 2.48(\mathrm{~s}, 3 \mathrm{H}), 2.41(\mathrm{~s}, 3 \mathrm{H}) .{ }^{13} \mathrm{C}$ 
573 574 575 576 577 578 579 580 581 415.1181.

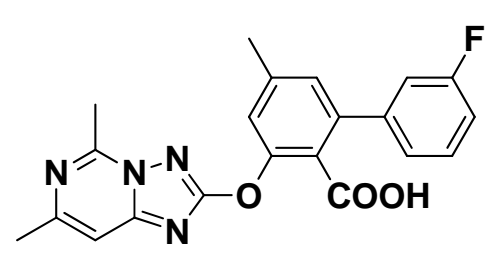

582 583 584 585 586 587

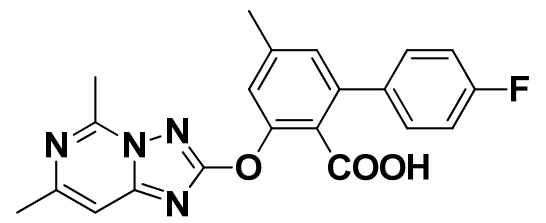

5-51

NMR (125 MHz, DMSO-d 6 ) : 168.71, 167.01, 154.97, 152.11, 150.42, 149.05, 146.93, $146.14,140.97,138.18,129.55,127.43,124.28,123.55,121.62,105.78,23.43,20.76,19.24$. HRMS (MALDI): $[\mathrm{M}+\mathrm{H}]^{+}$calcd for $\mathrm{C}_{21} \mathrm{H}_{17} \mathrm{~N}_{5} \mathrm{O}_{5}, 420.1308$; found, 420.1309.

Data for 3-((5,7-Dimethyl-[1,2,4]triazolo[1,5-c]pyrimidin-2-yl)oxy)-4 ' -fluoro-5methyl-[1,1' -biphenyl]-2-carboxylic Acid (5-51). White solid, 33\% yield, mp: 220-221 ${ }^{\circ} \mathrm{C}$. ${ }^{1} \mathrm{H}$ NMR (400 M Hz, DMSO-d $\left.d_{6} \delta\right): 13.12(\mathrm{~s}, 1 \mathrm{H}), 7.47(\mathrm{~d}, J=12.0 \mathrm{~Hz}, 3 \mathrm{H}), 7.28(\mathrm{dd}, J=$ 16.0, 7.2 Hz, 3H), 7.15 (s, 1H), 2.77 (s, 3H), $2.48(\mathrm{~s}, 3 \mathrm{H}), 2.38(\mathrm{~s}, 3 \mathrm{H}) .{ }^{13} \mathrm{C} \mathrm{NMR}(125 \mathrm{MHz}$, DMSO- $\left._{6} \delta\right): 168.72,167.38,162.85,160.90,154.91,152.10,150.15,149.04,140.36$, $138.98,135.74,135.72,130.20,130.14,127.36,124.37,120.33,115.37,115.20,105.76$, 23.42, 20.77, 19.25. HRMS (MALDI): $[\mathrm{M}+\mathrm{Na}]^{+}$calcd for $\mathrm{C}_{21} \mathrm{H}_{17} \mathrm{FN}_{4} \mathrm{O}_{3}, 415.1182$; found,

Data for 3-((5,7-Dimethyl-[1,2,4]triazolo[1,5-c]pyrimidin-2-yl)oxy)-3 ' -fluoro-5methyl-[1,1' -biphenyl]-2-carboxylic Acid (5-52). White solid, 30\% yield, mp: $217-218{ }^{\circ} \mathrm{C}$. ${ }^{1} \mathrm{H}$ NMR (600 M Hz, DMSO- $d_{6} \delta$ ): $13.20(\mathrm{~s}, 1 \mathrm{H}), 7.53-7.48(\mathrm{~m}, 1 \mathrm{H}), 7.47(\mathrm{~s}, 1 \mathrm{H}), 7.28(\mathrm{~d}$, $J=7.2 \mathrm{~Hz}, 3 \mathrm{H}), 7.24(\mathrm{~d}, J=9.0 \mathrm{~Hz}, 1 \mathrm{H}), 7.20(\mathrm{~s}, 1 \mathrm{H}), 2.77(\mathrm{~s}, 3 \mathrm{H}), 2.48(\mathrm{~s}, 3 \mathrm{H}), 2.39$ (s, 3H). ${ }^{13} \mathrm{C}$ NMR (125 MHz, DMSO-d $\left.d_{6} \delta\right): 168.68,167.27,162.89,160.95,154.92,152.10$, 
$590+\mathrm{H}]^{+}$calcd for $\mathrm{C}_{21} \mathrm{H}_{17} \mathrm{FN}_{4} \mathrm{O}_{3}, 393.1363$; found, 393.1366 .

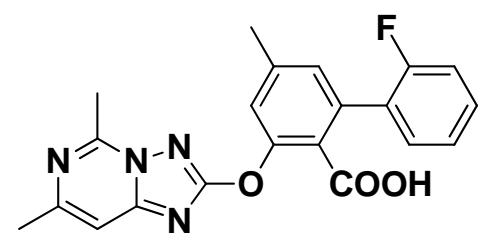

591

5-53

592

Data for 3-((5,7-Dimethyl-[1,2,4]triazolo[1,5-c]pyrimidin-2-yl)oxy)-2 ' -fluoro-5593 methyl-[1,1' -biphenyl]-2-carboxylic Acid (5-53). White solid, 20\% yield, mp: $211-212{ }^{\circ} \mathrm{C}$. $594{ }^{1} \mathrm{H}$ NMR (400 M Hz, DMSO-d $6 \delta$ ): $12.90(\mathrm{~s}, 1 \mathrm{H}), 7.48-7.39(\mathrm{~m}, 2 \mathrm{H}), 7.37(\mathrm{~d}, J=8.0 \mathrm{~Hz}$, $5951 \mathrm{H}), 7.29(\mathrm{~s}, 1 \mathrm{H}), 7.27(\mathrm{~s}, 1 \mathrm{H}), 7.25(\mathrm{~s}, 1 \mathrm{H}), 7.14(\mathrm{~s}, 1 \mathrm{H}), 2.77(\mathrm{~s}, 3 \mathrm{H}), 2.48(\mathrm{~s}, 3 \mathrm{H}), 2.38(\mathrm{~s}$ $5963 \mathrm{H}) .{ }^{13} \mathrm{C}$ NMR (125 MHz, DMSO-d $\left.d_{6} \delta\right): 168.82,166.62,159.89,157.94,154.91,152.10$, $597150.52,149.02,140.72,134.79,131.05,129.99,129.93,128.37,127.15,127.03,124.69$, 598 124.32, 124.30, 121.33, 115.50, 115.32, 105.74, 23.43, 20.71, 19.25. HRMS (MALDI): [M $599+\mathrm{H}]^{+}$calcd for $\mathrm{C}_{21} \mathrm{H}_{17} \mathrm{FN}_{4} \mathrm{O}_{3}, 393.1363$; found, 393.1360.

600

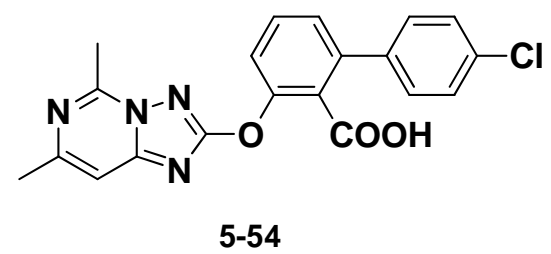

601 Data for 4' -Chloro-3-((5,7-dimethyl-[1,2,4]triazolo[1,5-c]-pyrimidin-2-yl)oxy)-[1,1'

602 -biphenyl]-2-carboxylic Acid (5-54). White solid, 29\% yield, mp: $224-225{ }^{\circ} \mathrm{C}$. ${ }^{1} \mathrm{H}$ NMR 603 (400 M Hz, DMSO- $\left.d_{6} \delta\right): 13.34(\mathrm{~s}, 1 \mathrm{H}), 7.59$ (t, $\left.J=8.0 \mathrm{~Hz}, 1 \mathrm{H}\right), 7.55(\mathrm{~s}, 1 \mathrm{H}), 7.53(\mathrm{~s}, 1 \mathrm{H})$, $6047.49(\mathrm{~d}, J=2.4 \mathrm{~Hz}, 2 \mathrm{H}), 7.46(\mathrm{~s}, 2 \mathrm{H}), 7.35(\mathrm{~d}, J=7.6 \mathrm{~Hz}, 1 \mathrm{H}), 2.77(\mathrm{~s}, 3 \mathrm{H}), 2.48(\mathrm{~s}, 3 \mathrm{H})$. $605{ }^{13} \mathrm{C}$ NMR (125 MHz, DMSO-d 6 ): 168.62, 167.16, 154.97, 152.10, 150.02, 149.07, 
$606138.81,137.95,132.85,130.29,129.99,128.51,127.08,126.52,120.35,105.76,23.43$,

607 19.24. HRMS (MALDI): $[\mathrm{M}+\mathrm{H}]^{+}$calcd for $\mathrm{C}_{20} \mathrm{H}_{15} \mathrm{ClN}_{4} \mathrm{O}_{3}, 395.0911$; found, 395.0912 .

608

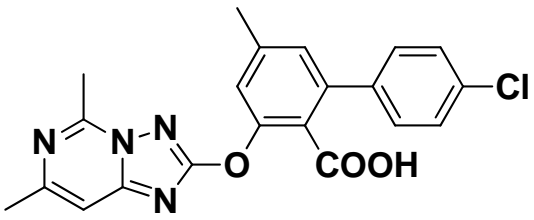

5-55

609 Data for 4' -Chloro-3-((5,7-dimethyl-[1,2,4]triazolo[1,5-c]-pyrimidin-2-yl)oxy)-5-

610 methyl-[1,1' -biphenyl]-2-carboxylic Acid (5-55). White solid, 48\% yield, mp: $224-225{ }^{\circ} \mathrm{C}$.

$611{ }^{1} \mathrm{H}$ NMR $\left(400 \mathrm{MHz}, \mathrm{DMSO}_{-} d_{6} \delta\right): 13.17(\mathrm{~s}, 1 \mathrm{H}), 7.52(\mathrm{~d}, J=8.4 \mathrm{~Hz}, 2 \mathrm{H}), 7.45(\mathrm{~d}, J=8.4$

$612 \mathrm{~Hz}, 3 \mathrm{H}), 7.27(\mathrm{~s}, 1 \mathrm{H}), 7.17(\mathrm{~s}, 1 \mathrm{H}), 2.77(\mathrm{~s}, 3 \mathrm{H}), 2.48(\mathrm{~s}, 3 \mathrm{H}), 2.38(\mathrm{~s}, 3 \mathrm{H}) .{ }^{13} \mathrm{C}$ NMR $(125$

$613 \mathrm{MHz}, \mathrm{DMSO}^{-} d_{6} \delta$ ): 168.73, 167.29, 154.92, 152.09, 150.22, 149.02, 140.51, 138.81,

$614138.20,132.72,129.94,128.43,127.27,124.29,120.62,105.76,23.42,20.77,19.25$.

615 HRMS (MALDI): $[\mathrm{M}+\mathrm{H}]^{+}$calcd for $\mathrm{C}_{21} \mathrm{H}_{17} \mathrm{ClN}_{4} \mathrm{O}_{3}, 409.1067$; found, 409.1071 .

616

617

618

619

620

621

622

623

624

625 
$626{ }^{1} \mathrm{H}$ NMR and ${ }^{13} \mathrm{C}$ NMR spectral data for representative compounds
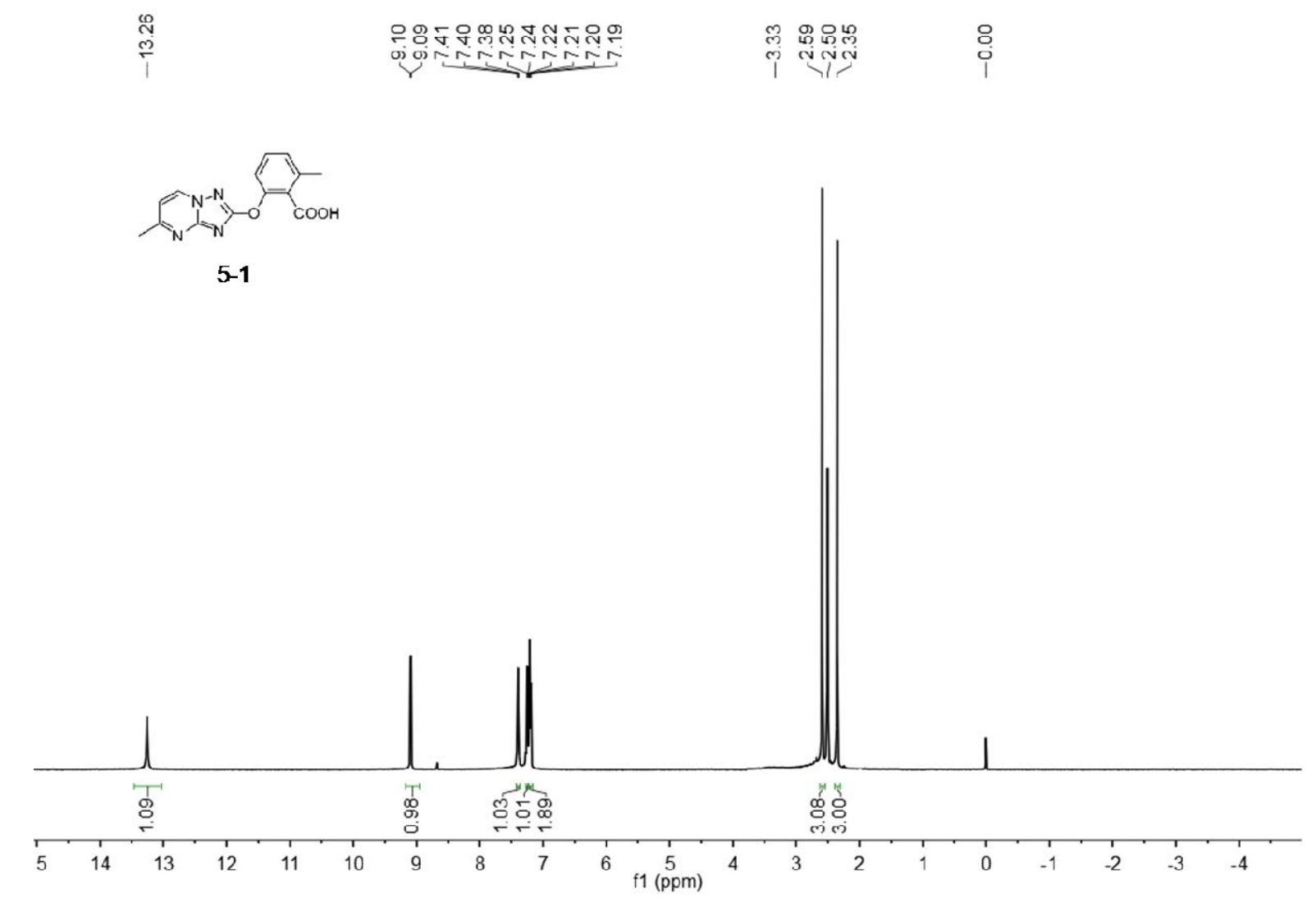

628
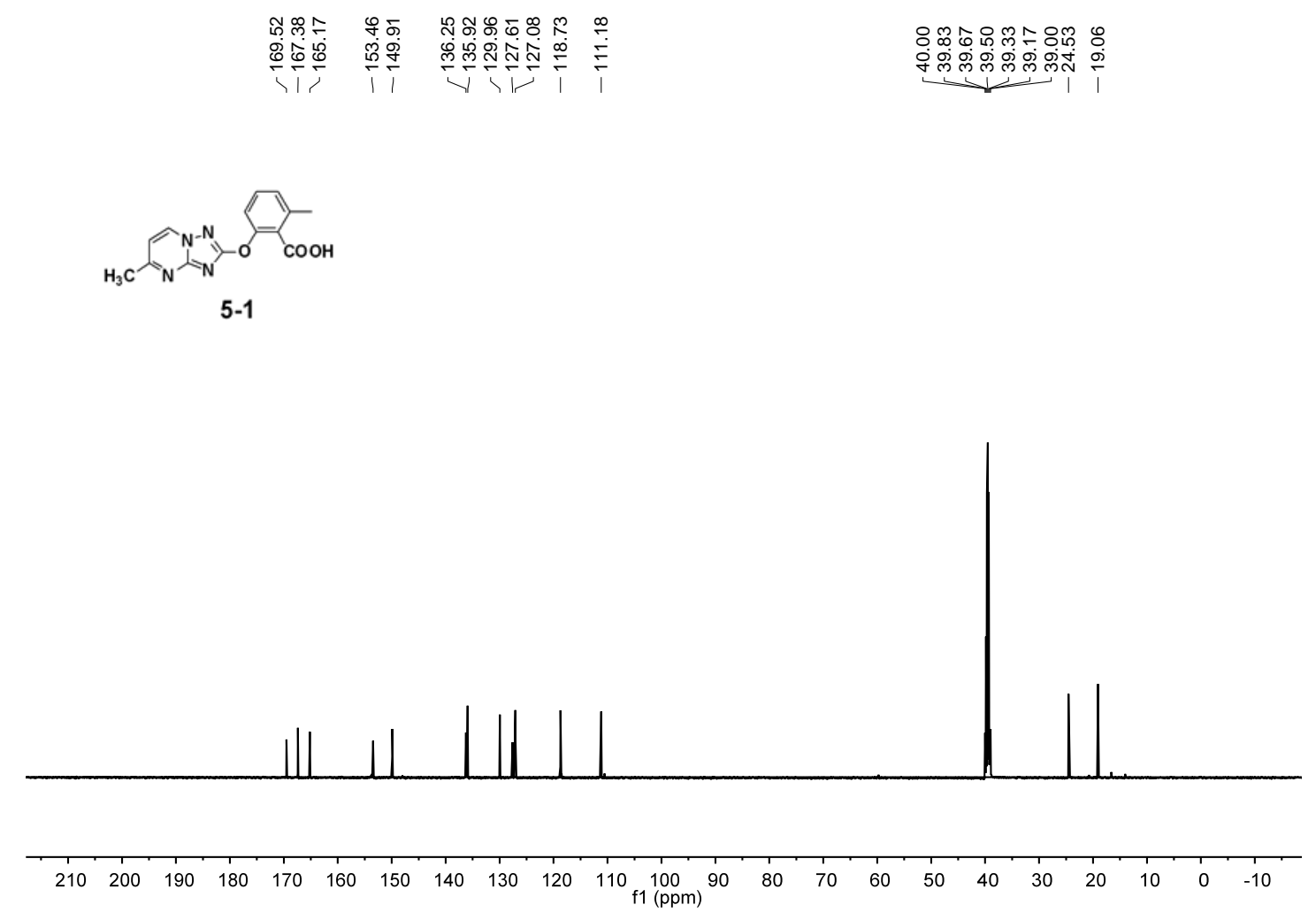

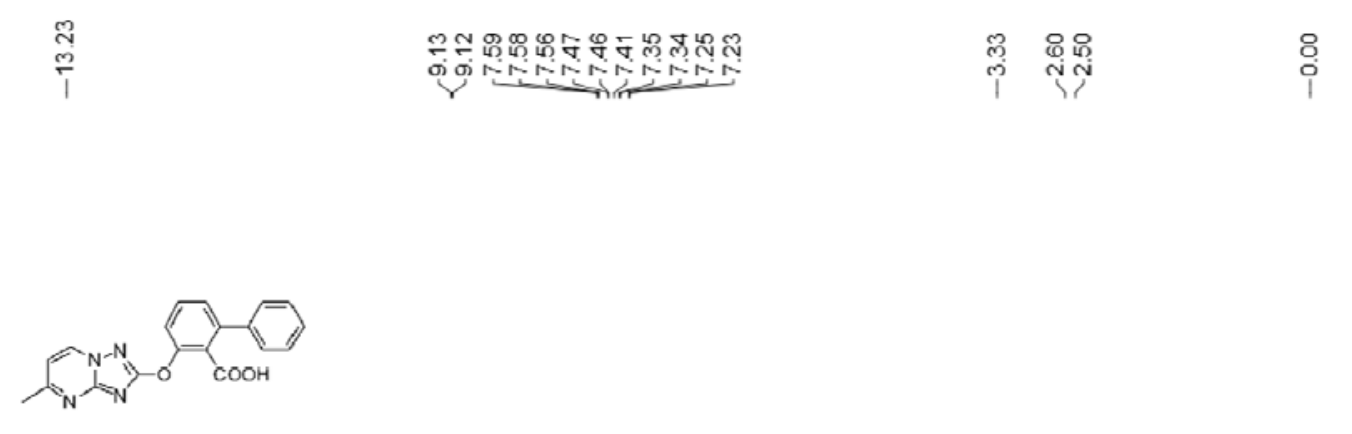

5-2

630

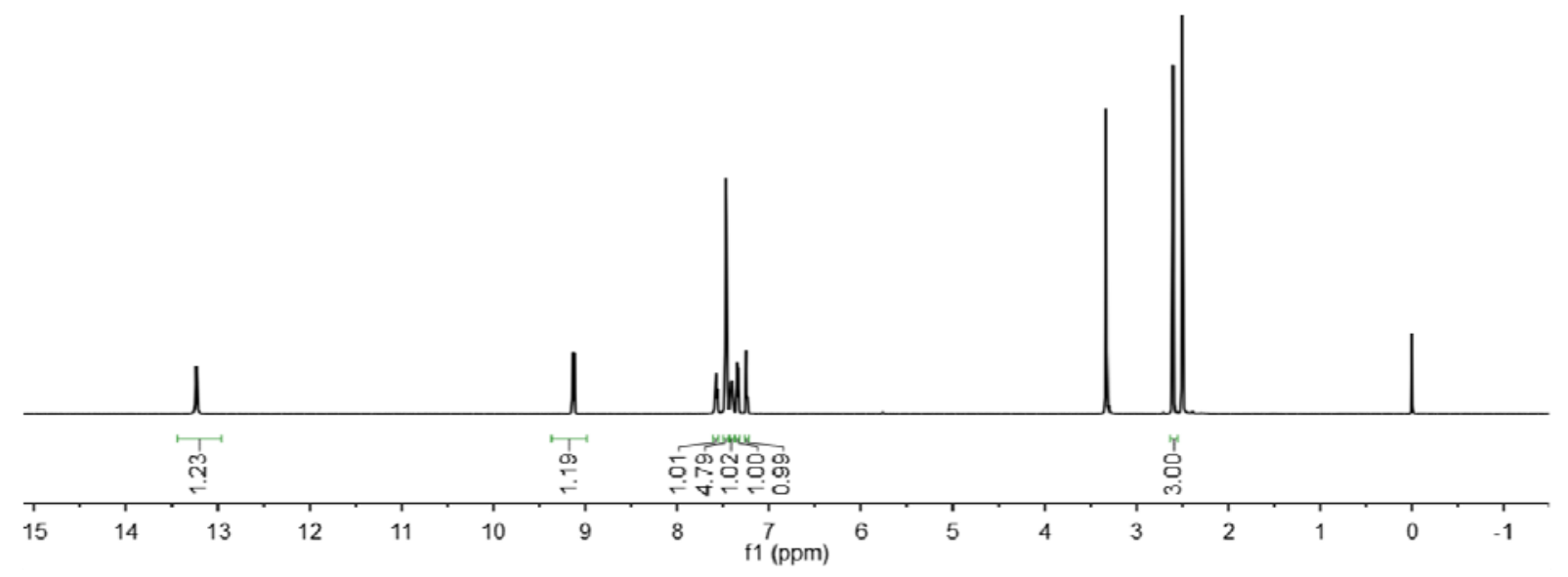

631

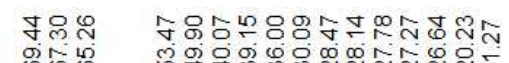

ڤัต

iद

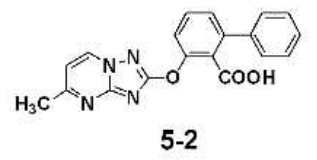

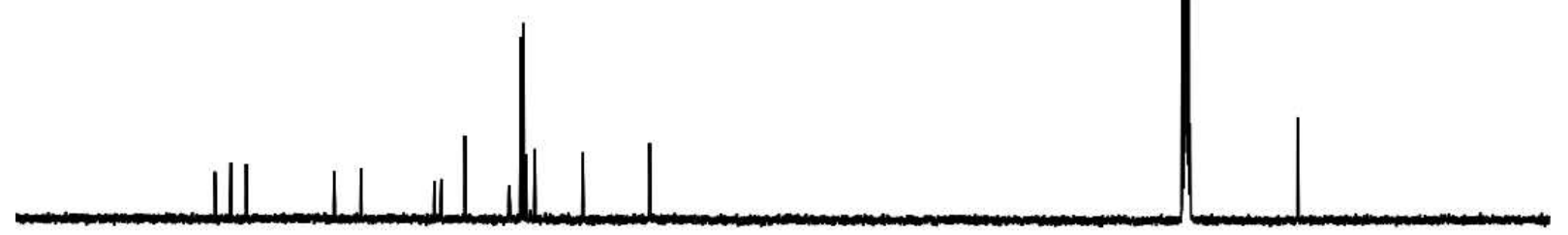




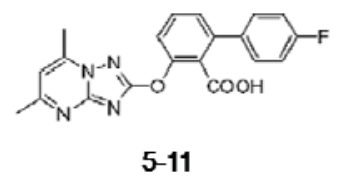

633

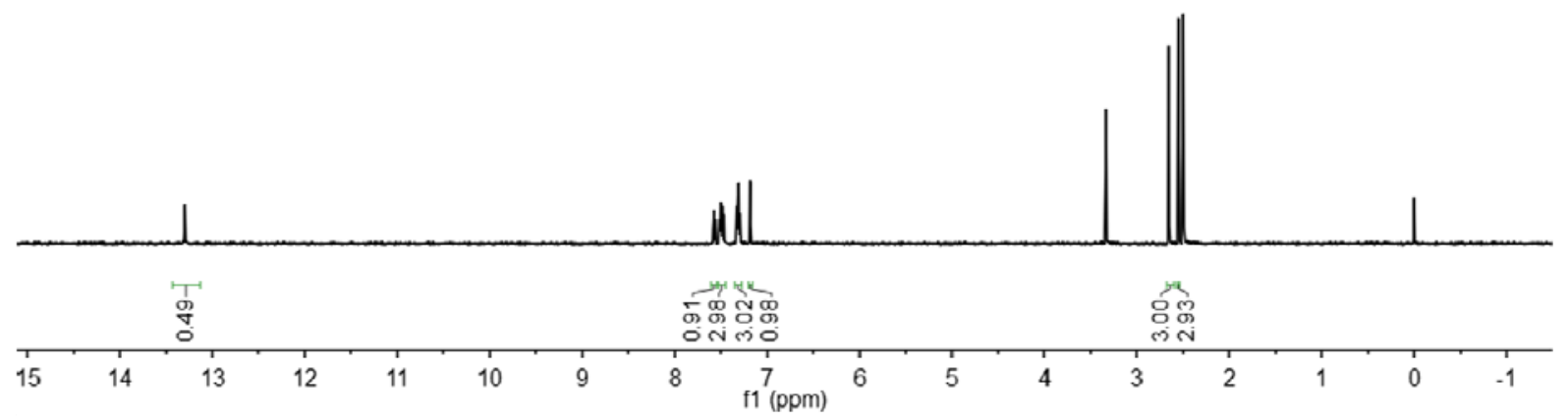

634

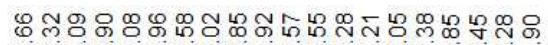

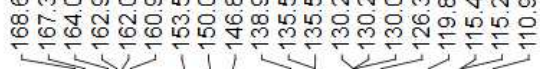

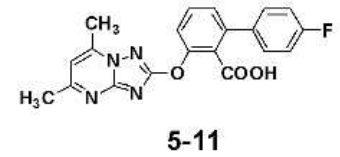

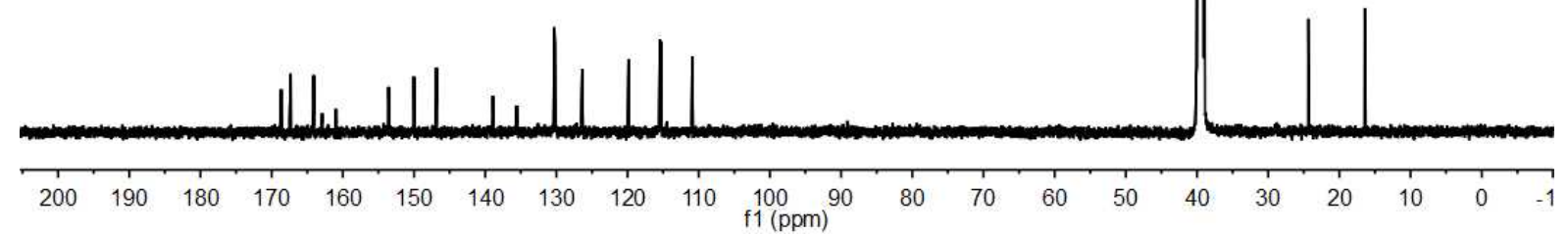



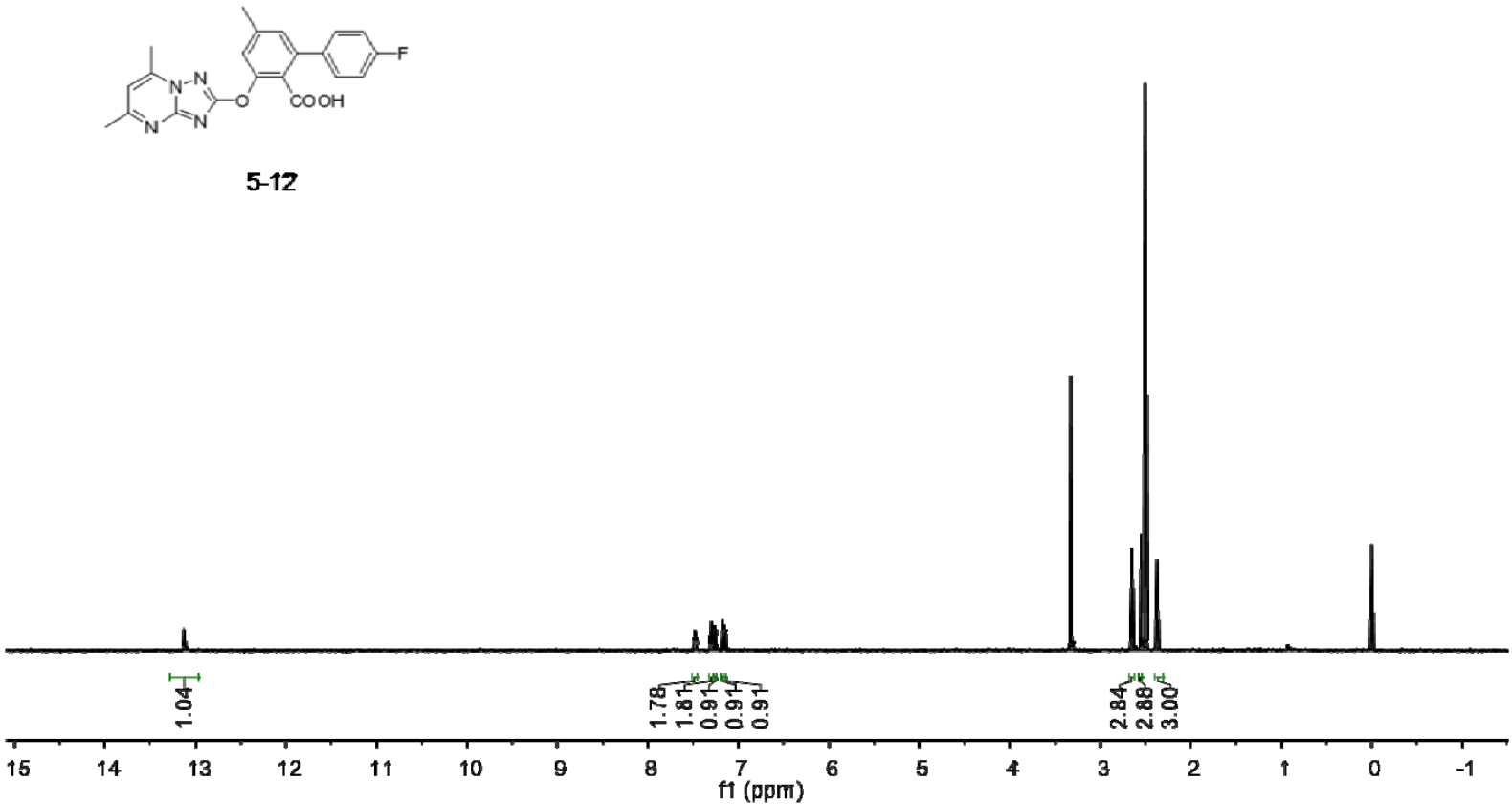

637

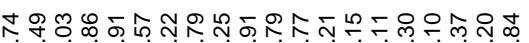

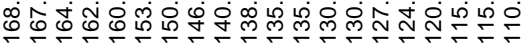

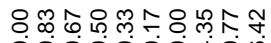

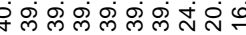

(

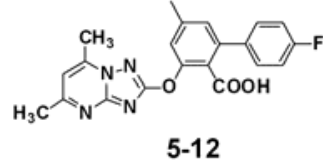




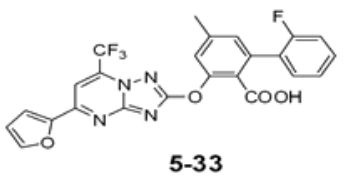

640

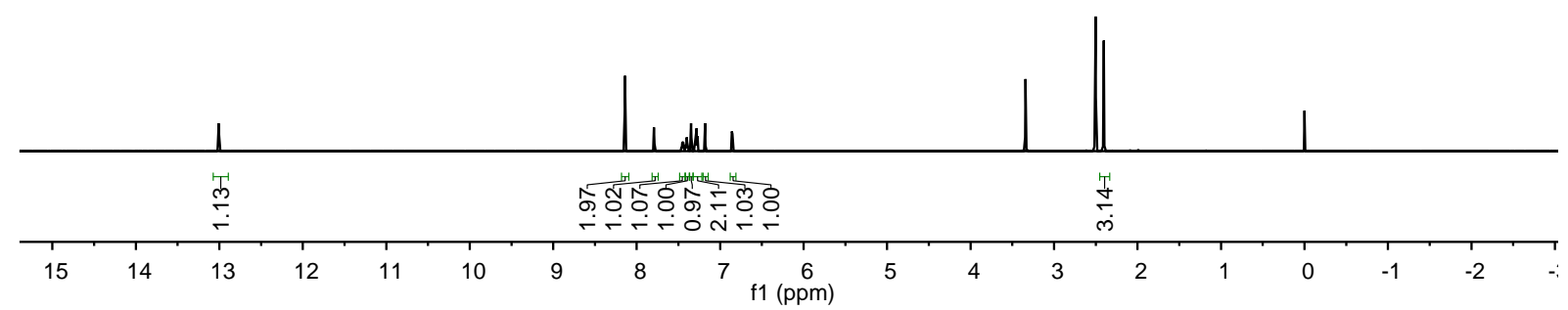

641

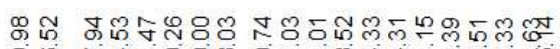

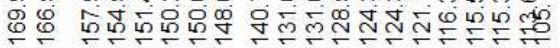

1
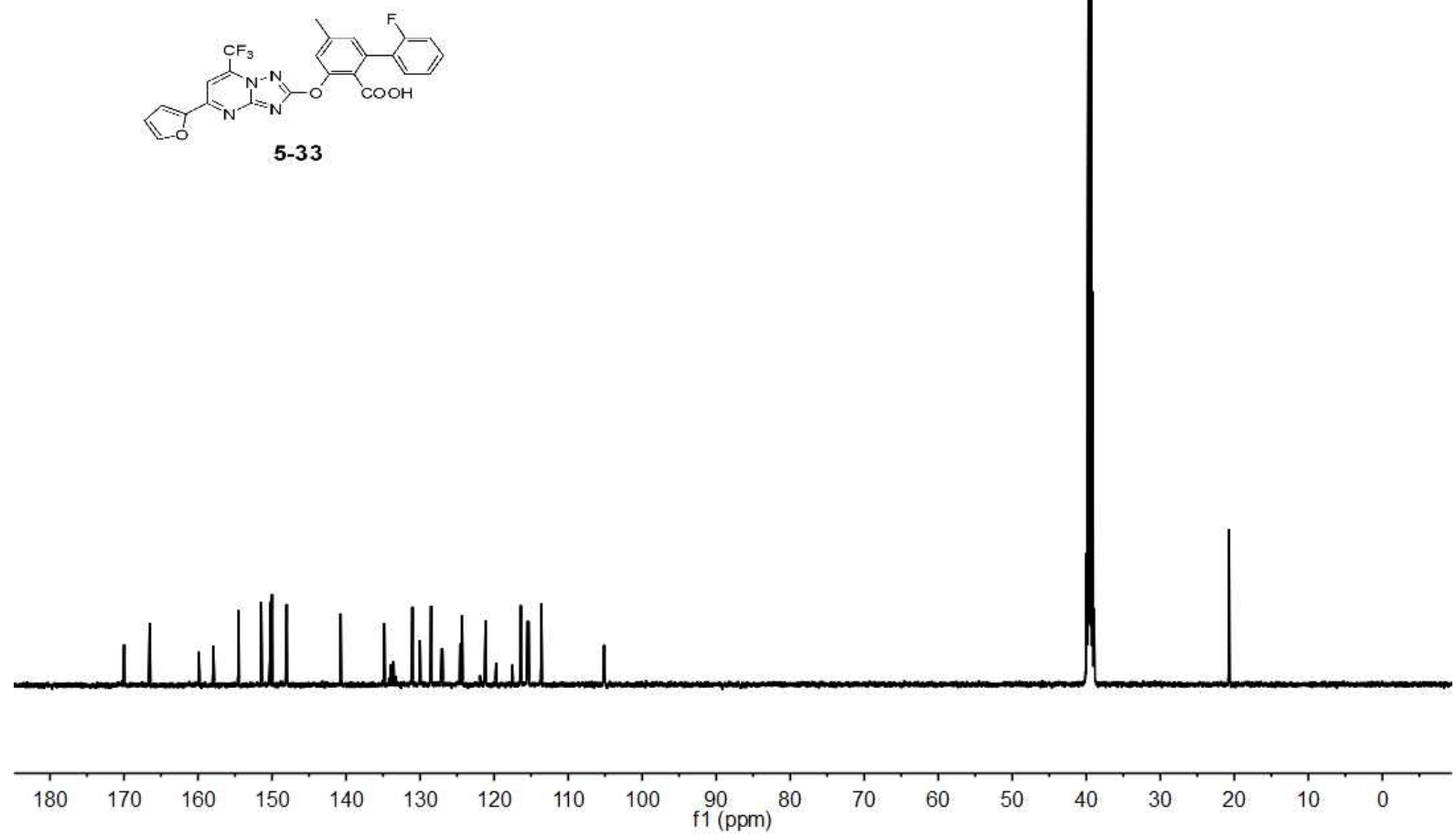


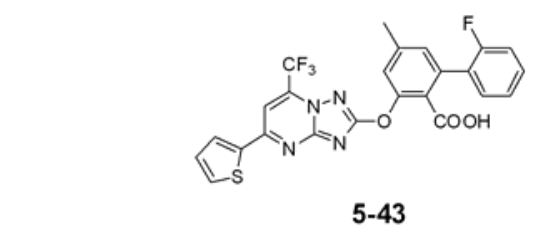

644

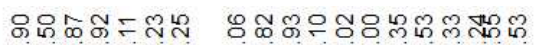

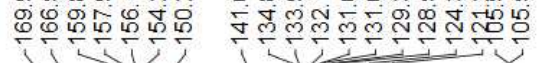
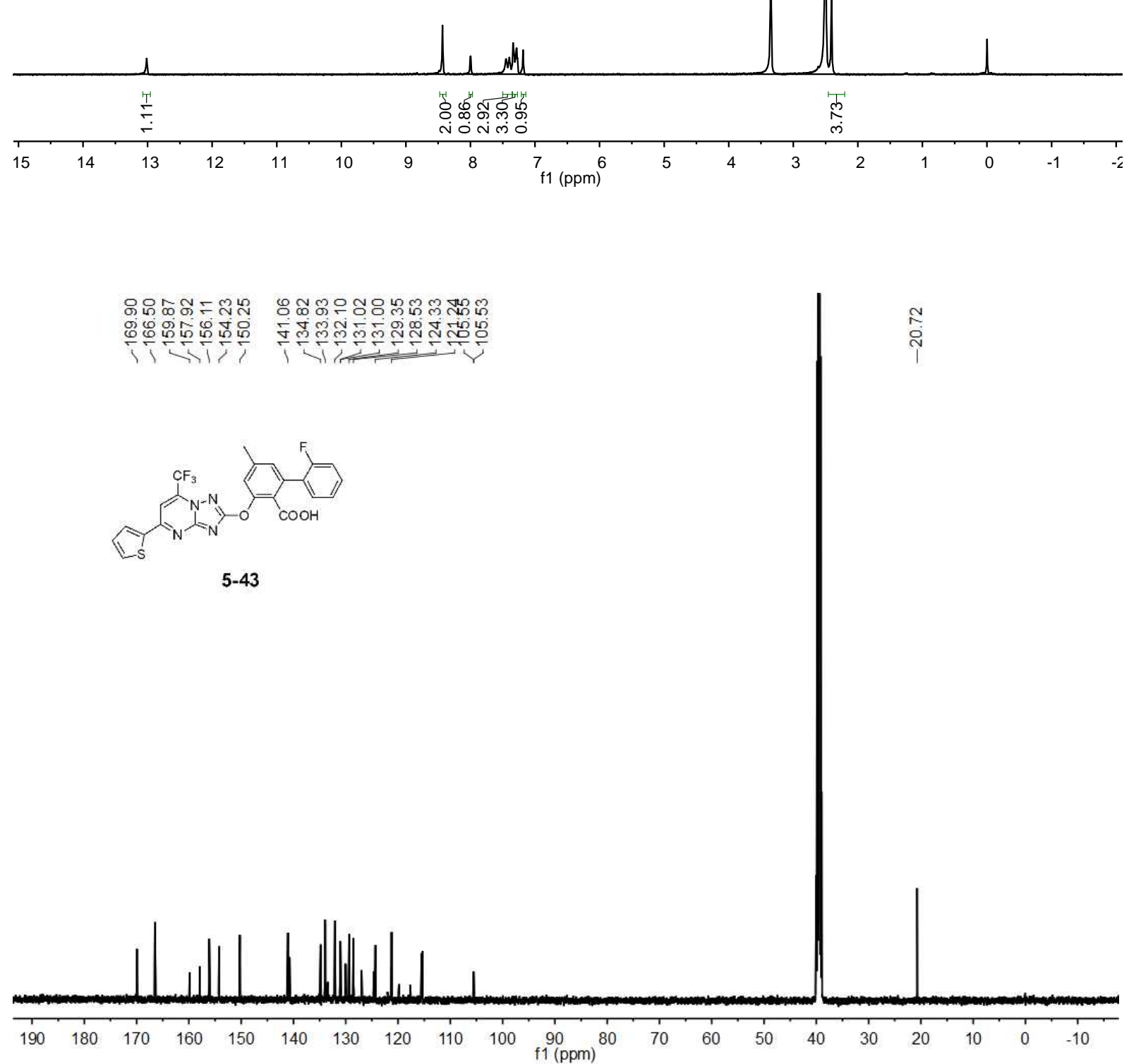

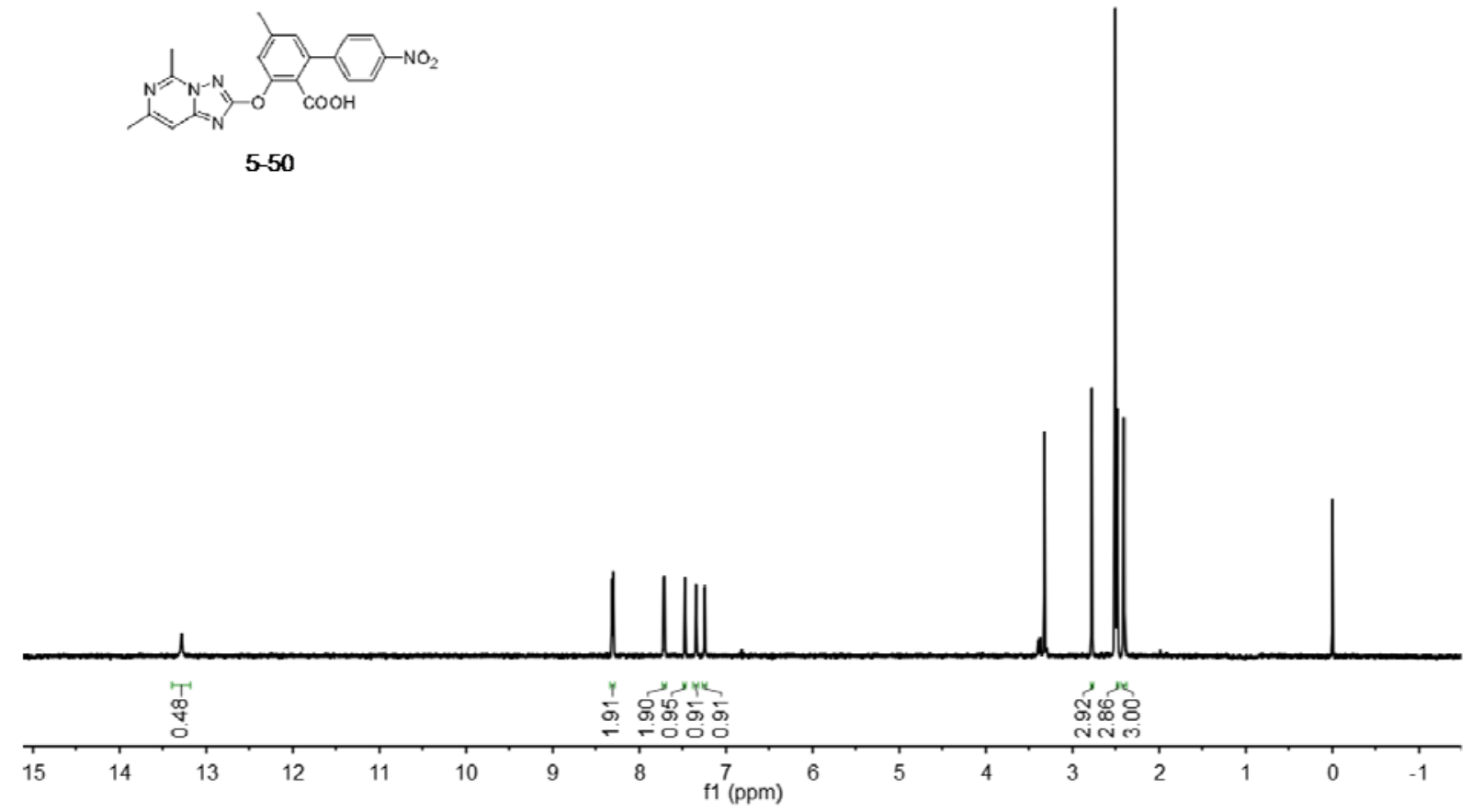

647

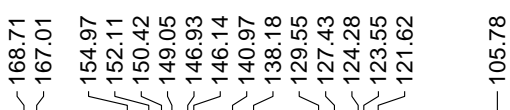

В ๓ ํํㅇำ

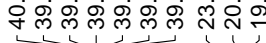
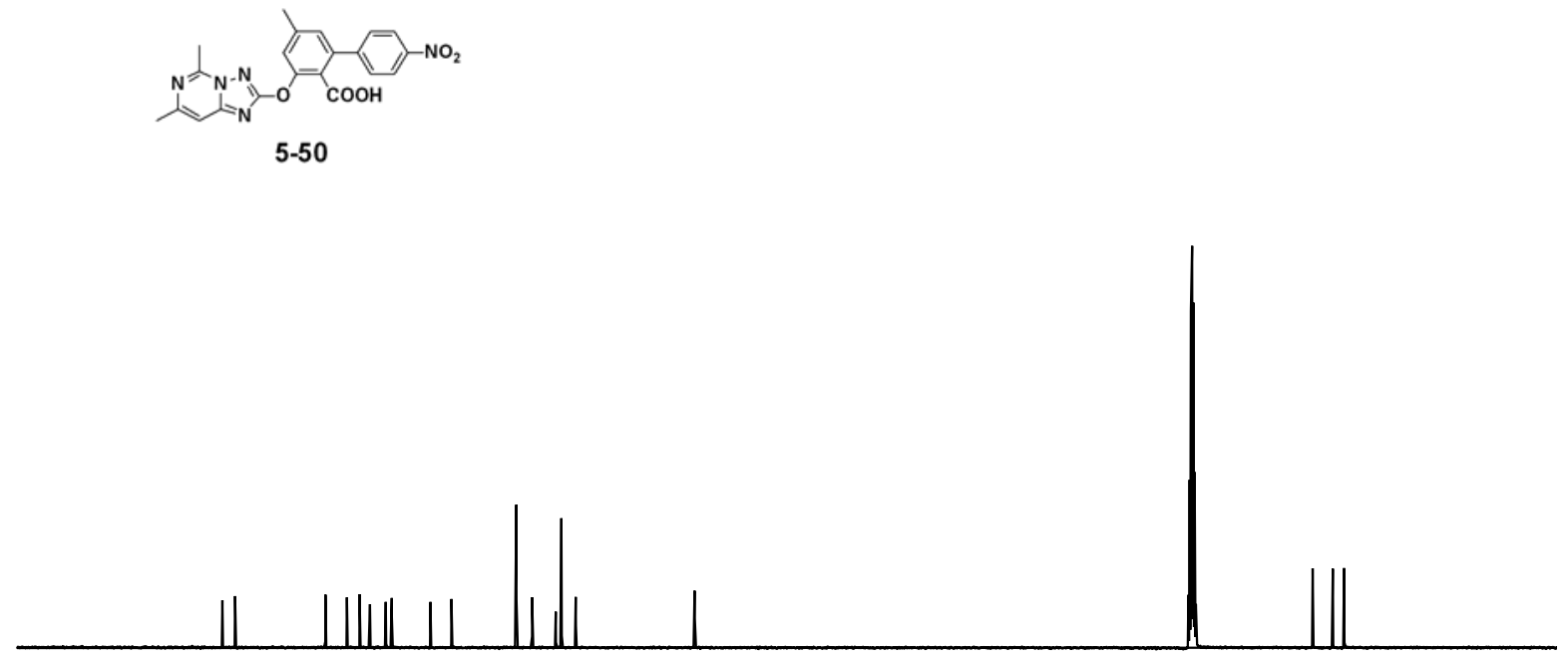
651 1. Yang, G. F.; Lu, R. J.; Fei, X. N.; Yang, H. Z. Syntheses and Properties of New 652 Herbicidal 2-Arylthio-1,2,4- Triazolo[1,5-a]- pyrimidine Derivatives. Chinese J. Chem. 2000, $18,435-440$.

2. Liu, Y. C.; Huang, Z. Y.; Chen, Q.; Yang, G. F. Efficient synthesis of functionalized 6arylsalicylates via microwave-promoted Suzuki cross-coupling reaction. Tetrahedron 2013, 69, 9025-9032.

3. Liu, Y. C.; Qu, R. Y.; Chen, Q.; Wu, Q. Y.; Yang, G. F., Efficient synthesis of functionalized 6-substituted-thiosalicylates via microwave-promoted Suzuki crosscoupling reaction. Tetrahedron 2014, 70, 2746-2752.

4. Qu, R. Y.; Liu, Y. C.; Wu, Q. Y.; Chen, Q.; Yang, G. F. An efficient method for syntheses of functionalized 6-bulkysubstituted salicylates under microwave irradiation. Tetrahedron 2015, 71, 8123-8130.

5. Yawer, M. A.; Hussain, I.; Iqbal, I.; Spannenberg, A.; Langer, P., Synthesis of functionalized $6(5 \mathrm{H})$-phenanthridinones based on a [3+3]-cyclocondensation/ lactamization strategy. Tetrahedron Lett. 2008, 49, 4467-4469.

6. Hussain, I.; Yawer, M. A.; Lau, M.; Pundt, T.; Fischer, C.; Görls, H.; Langer, P., 667 Regioselective Synthesis of Fluorinated Phenols, Biaryls, 6H - Benzo [c] chromen-6ones and Fluorenones Based on Formal [3+3] Cyclizations of 1, 3-Bis (silyl enol ethers). Eur. J. Org. Chem. 2008, 2008, 503-518.

7. Hussain, I.; Nguyen, V. T. H.; Yawer, M. A.; Dang, T. T.; Fischer, C.; Reinke, H.; Langer, P., Synthesis of Dibenzo [b, d] pyran-6-ones Based on [3+ 3] Cyclizations of 1, 
672 3-Bis (silyl enol ethers) with 3-Silyloxy-2-en-1-ones. J. Org. Chem. 2007, 72, 6255$673 \quad 6258$.

674

675 